\title{
Spherical collapse and the halo model in braneworld gravity
}

\author{
Fabian Schmidt, ${ }^{1}$ Wayne $\mathrm{Hu}^{2}$ and Marcos Lima ${ }^{3}$ \\ ${ }^{1}$ Theoretical Astrophysics, California Institute of Technology M/C 350-17, Pasadena, California 91125-0001, USA \\ ${ }^{2}$ Department of Astronomy and Astrophysics, Kavli Institute for Cosmological Physics, and Enrico Fermi Institute, \\ The University of Chicago, Chicago, Illinois 60637-1433, USA \\ ${ }^{3}$ Department of Physics and Astronomy, University of Pennsylvania, Philadelphia, Pennsylvania 19104, USA
}

(Received 30 November 2009; published 15 March 2010)

We present a detailed study of the collapse of a spherical perturbation in Dvali-Gabadadze-Porrati (DGP) braneworld gravity for the purpose of modeling simulation results for the halo mass function, bias, and matter power spectrum. The presence of evolving modifications to the gravitational force in the form of the scalar brane-bending mode leads to qualitative differences to the collapse in ordinary gravity. In particular, differences in the energetics of the collapse necessitate a new, generalized method for defining the virial radius which does not rely on strict energy conservation. These differences and techniques apply to smooth dark energy models with $w \neq-1$ as well. We also discuss the impact of the exterior of the perturbation on collapse quantities due to the lack of a Birkhoff theorem in DGP. The resulting predictions for the mass function, halo bias, and power spectrum are in good overall agreement with DGP $N$-body simulations on both the self-accelerating and normal branch. In particular, the impact of the Vainshtein mechanism as measured in the full simulations is matched well. The model and techniques introduced here can serve as practical tools for placing consistent constraints on braneworld models using observations of large-scale structure.

DOI: 10.1103/PhysRevD.81.063005

PACS numbers: 95.30.Sf, 95.36.+x, 98.80.-k, 98.80.Jk

\section{INTRODUCTION}

Modified gravity models have attracted a great deal of interest recently as an alternative explanation of the observed accelerated expansion of the Universe [1-5]. In order for this scenario to work, gravity must be significantly modified from general relativity (GR) on cosmological scales, but has to reduce to GR locally in order to satisfy stringent solar system constraints at a few AU. Thus, a working modified gravity model has to include a nonlinear mechanism to restore GR in high-density environments, which can have a noticeable impact on the formation of large-scale structure on intermediate scales of a few to tens of Mpc [6-9].

One popular modified gravity scenario is the DvaliGabadadze-Porrati (DGP) braneworld model [10]. Here, a four-dimensional Friedmann-Robertson-Walker universe is imbedded as a brane in five-dimensional Minkowski space. In this model, gravity is five-dimensional on the largest scales, and becomes four-dimensional at scales below the crossover scale $r_{c}$, a fundamental parameter of the model. The modification of the Friedmann equation depends on the choice of embedding for the brane [11] which yields two branches of the model: the selfaccelerating branch, on which accelerated expansion occurs at late times without any cosmological constant or dark energy, and the normal branch where no such acceleration occurs, and a brane tension or other form of stressenergy with negative pressure has to be added on the brane.

On scales much smaller than the horizon and crossover scales, DGP gravity can be described by an effective scalar-tensor theory $[12,13]$, where the additional scalar degree of freedom, the brane-bending mode $\varphi$ is associated with displacements of the brane from its background position. The brane-bending mode yields an additional gravitational force which influences dynamics of nonrelativistic particles. On the normal branch of DGP, this force is attractive, while it is repulsive in the self-accelerating branch. Nonlinear interactions of $\varphi$, via the so-called Vainshtein mechanism, ensure that it has tiny effects within the Solar System. For values of $r_{c}$ of order the current horizon, these interactions become important as soon as the density contrast becomes of order unity. Hence, it is crucial to consistently follow the full brane-bending mode interactions in a cosmological simulation, as has recently been done $[9,14]$. While we study the behavior of $\varphi$ in specific DGP models, the form of the nonlinear interactions is expected to be generic to braneworld models with large extra dimensions $[15,16]$.

Given the considerable computational expense of these simulations, it is worthwhile to develop a model which captures the main modified gravity effects, enabling forecasts and constraints properly marginalized over cosmological parameters. In the halo model of large structure [17], which assumes that all matter is in bound dark matter halos, the abundance and clustering of halos are determined by the linear power spectrum once characteristic quantities of the collapse of a spherical perturbation are determined, namely, the linear collapse threshold and the virial radius or overdensity.

Spherical collapse is well studied for general relativity with a cosmological constant (e.g., [18,19]), and has also been explored for quintessence-type dark energy [20-22]. 
A first study of spherical perturbations in the context of DGP was undertaken in [6]. We extend previous studies by deriving the full $\varphi$ field profile of an isolated mass in closed form, and by carefully defining the interior and exterior forces during collapse along with the energetics implied by the profile. In particular, the potential energy required for the virial theorem and for the total Newtonian energy differ, with the latter not being strictly conserved during collapse. ${ }^{1}$ This lack of a conservation law also applies to dark energy models with an equation of state $w \neq-1$. We show how this problem can be circumvented by properly defining the condition for virial equilibrium based on forces.

We discuss the general properties and parametrization of DGP models in Sec. II, and the spherical collapse calculation in Sec. III. The halo model calculations are outlined in Sec. IV, and the results and comparisons with simulations are given in Sec. V. We conclude in Sec. VI. The Appendix contains derivations of the $\varphi$ profile and other quantities needed in the collapse calculation, as well as a discussion of the potential energy and virial theorem in DGP.

\section{DGP MODELS}

In this section, we discuss the general properties of the DGP models considered in this paper. The first model (sDGP) is in the self-accelerating branch of DGP with neither a cosmological constant nor spatial curvature. During matter domination and beyond, the modified Friedmann equation in sDGP reads

$$
H_{\mathrm{sDGP}}(a)=H_{0}\left(\sqrt{\Omega_{\mathrm{rc}}}+\sqrt{\Omega_{m} a^{-3}+\Omega_{\mathrm{rc}}}\right),
$$

where

$$
\Omega_{\mathrm{rc}} \equiv \frac{1}{4 H_{0}^{2} r_{c}^{2}}, \quad \Omega_{m} \equiv \frac{8 \pi G}{3 H_{0}^{2}} \bar{\rho}_{0},
$$

and $\bar{\rho}_{0}$ is the average matter density today. This expansion history is clearly different from Lambda cold dark matter $(\Lambda \mathrm{CDM})$ and corresponds to an effective dark energy with $w_{\text {eff }} \rightarrow-1 / 2$ in the matter-dominated era at high redshifts. For comparison, we will also consider an effective smooth dark energy model (QCDM) with the same expansion history as sDGP. Note that this expansion history, when combined with the growth of structure near the horizon, is in substantial conflict with data [23]. Moreover, the selfaccelerating branch is plagued by ghost issues [12,24,25] when perturbed around the de Sitter limit. Despite these problems, sDGP remains an interesting toy model for acceleration from modified gravity.

\footnotetext{
${ }^{1}$ The "total energy" here is defined for a Newtonian cosmology, and its nonconservation does not imply violation of covariant energy-momentum conservation.
}

The second scenario (nDGP) is in the normal branch of DGP. In order to achieve acceleration, it is necessary to add a stress-energy component with negative pressure on the brane. We adopt the model introduced in [26], where a general dark energy component is added on the brane, but the geometry remains spatially flat. The equation of state of this dark energy is adjusted so that the expansion history is precisely $\Lambda \mathrm{CDM}$ :

$$
H_{\mathrm{nDGP}}(a)=H_{0} \sqrt{\Omega_{m} a^{-3}+\Omega_{\Lambda}},
$$

again during matter domination and beyond. While $\Omega_{m}$ quantifies the true matter content in this model, $\Omega_{\Lambda}$ is to be seen as an effective cosmological constant relevant for the expansion history only. This construction allows our nDGP models to evade the otherwise stringent expansion history constraints on $r_{c}$ with a true cosmological constant or brane tension [27]. Likewise, it provides a class of models where the observable impact of force modification is cleanly separated from the background geometry. We consider the two models of [26], nDGP-1 with $r_{c}=500 \mathrm{Mpc}$ and nDGP-2 with $r_{c}=3000 \mathrm{Mpc}$.

For notational simplicity, it is convenient to also phrase the sDGP Friedmann equation in terms of an effective dark energy component so that in both cases

$$
H^{2}=\frac{8 \pi G}{3}\left(\bar{\rho}+\rho_{\mathrm{eff}}\right)
$$

where $\bar{\rho}$ is the background matter density and $\rho_{\text {eff }}$ is implicitly defined by Eqs. (1) and (3). In Table I, we summarize the parameter choices for the simulated models $[9,26]$; in case of sDGP, they are from the best-fitting flat self-accelerating model of [23] to WMAP $5 \mathrm{yr}$, supernova, and $H_{0}$ data, while for the nDGP models, the expansion history and primordial normalization match those of the best-fitting $\Lambda$ CDM model of [23].

On scales much smaller than both the horizon $H^{-1}$ and the crossover scale $r_{c}$, DGP reduces to an effective scalar-

TABLE I. Parameters of the simulated DGP cosmologies.

\begin{tabular}{lccccc}
\hline \hline & QCDM & sDGP & $\Lambda$ CDM & nDGP-1 & nDGP-2 \\
\hline$\Omega_{m}$ & 0.258 & 0.258 & 0.259 & 0.259 & 0.259 \\
$\Omega_{\Lambda}$ (eff.) & 0 & 0 & 0.741 & 0.741 & 0.741 \\
$r_{c}[\mathrm{Mpc}]$ & $\infty$ & 6118 & $\infty$ & 500 & 3000 \\
$\Omega_{\mathrm{rc}}$ & 0 & 0.138 & 0 & 17.5 & 0.487 \\
$H_{0}[\mathrm{~km} / \mathrm{s} / \mathrm{Mpc}]$ & 66.0 & 66.0 & 71.6 & 71.6 & 71.6 \\
$100 \Omega_{b} h^{2}$ & 2.37 & & 2.26 & & \\
$\Omega_{c} h^{2}$ & 0.089 & & 0.110 & & \\
$\tau$ & 0.0954 & 0.0825 & & \\
$n_{s}$ & 0.998 & 0.959 & & \\
$A_{s}\left(0.05 \mathrm{Mpc}^{-1}\right)$ & $2.01610^{-9}$ & $2.10710^{-9}$ & \\
$\sigma_{8}(\Lambda \mathrm{CDM})^{\mathrm{a}}$ & 0.6566 & 0.7892 & & \\
\hline \hline
\end{tabular}

${ }^{a}$ Linear power spectrum normalization today of a $\Lambda \mathrm{CDM}$ model with the same primordial normalization. 
tensor theory with the brane-bending mode $\varphi$ representing the scalar. Time variation in $\varphi$ induced by the nonrelativistic motion of the matter involve the dynamical time and can be neglected with respect to spatial derivatives in this regime. The $\varphi$ field then couples to matter by contributing to the metric potentials $\Psi, \Phi$ defined by the line element

$$
d s^{2}=-(1+2 \Psi) d t^{2}+a^{2}(1+2 \Phi) d x^{2}
$$

as

$$
\begin{gathered}
\Psi=\Psi_{N}+\frac{1}{2} \varphi, \\
\Phi=-\Psi_{N}+\frac{1}{2} \varphi,
\end{gathered}
$$

where $\Psi_{N}$ is the Newtonian potential determined via the usual Poisson equation

$$
\nabla^{2} \Psi_{N}=4 \pi G \delta \rho .
$$

Here and throughout, spatial derivatives are physical, not comoving.

While the motion of massive, nonrelativistic particles such as cold dark matter is governed by the dynamical potential $\Psi$, the propagation of light is determined by the lensing potential $(\Psi-\Phi) / 2$. This combination is not affected by $\varphi$ due to the conformal invariance of electromagnetism. Hence, in DGP lensing mass is equal to the "actual" mass, while the dynamical mass differs unless $\left|\varphi / \Psi_{N}\right| \ll 1$.

In the subhorizon, quasistatic regime, the equation for the brane-bending mode can be written as (e.g., [28])

$$
\nabla^{2} \varphi+\frac{r_{c}^{2}}{3 \beta}\left[\left(\nabla^{2} \varphi\right)^{2}-\left(\nabla_{i} \nabla_{j} \varphi\right)\left(\nabla^{i} \nabla^{j} \varphi\right)\right]=\frac{8 \pi G}{3 \beta} \delta \rho,
$$

where the function $\beta(a)$ is given by

$$
\beta(a)=1 \pm 2 H(a) r_{c}\left(1+\frac{\dot{H}(a)}{3 H^{2}(a)}\right) .
$$

Here, the positive sign holds for the normal branch of DGP, while the negative sign holds for the self-accelerating branch. Note that the sign of $\beta$ determines whether the force mediated by the brane-bending mode is attractive $(\beta>0, \mathrm{nDGP})$ or repulsive $(\beta<0, \mathrm{sDGP})$.

\section{TOP-HAT COLLAPSE IN DGP}

In this section, we review the dynamics of the collapse of a top-hat density perturbations in the DGP case. We follow $[6,8]$ but pay special attention to the $\varphi$ profile as well as subtleties in the potential energy and virial condition which are specific to the Vainshtein mechanism (see the Appendix for details).

We assume an initial top-hat density profile of the form

$$
\rho(r)-\bar{\rho}= \begin{cases}\delta \rho, & r \leq R \\ 0, & r>R\end{cases}
$$

We show in the Appendix that the top-hat profile remains top-hat during the collapse despite sweeping out an underdensity outside of $R$. We further show that forces inside of $R$ depend on the enclosed mass perturbation, and so we can ignore the impact of any compensating underdensity on the dynamics of collapse. Note that this is unique to a top-hat density and does not hold for other density profiles, in which case the collapse will not be self-similar anymore.

Given $\rho$ and $R$, there are two important mass parameters: the total mass $M=4 \pi \rho R^{3} / 3$ and the mass perturbation $\delta M=4 \pi \delta \rho R^{3} / 3$. The first is conserved during collapse, while the second is the source of the $\varphi$ field and gravitational potential, and is therefore useful in expressions involving the field profile. With the definition $\delta=\delta \rho / \bar{\rho}$, the two masses are related by

$$
\delta M=\frac{\delta}{1+\delta} M,
$$

such that they coincide at high overdensity.

\section{A. Collapse dynamics}

Given a metric specified by $\Psi, \Phi$, conservation of energy-momentum is unchanged in DGP and leads to the same equation of motion for the density perturbation as in ordinary gravity. On scales much smaller than the horizon

$$
\ddot{\delta}-\frac{4}{3} \frac{\dot{\delta}^{2}}{1+\delta}+2 H \dot{\delta}=(1+\delta) \nabla^{2} \Psi,
$$

where $H=\dot{a} / a$ denotes the Hubble rate, and dots denote derivatives with respect to time. The modification of gravity enters through the dynamical potential $\Psi$ : in GR, $\Psi=$ $\Psi_{N}$ [Eq. (8)], while in DGP $\Psi$ receives an additional contribution from the brane-bending mode $\varphi$ following Eq. (6).

The full profile of $\varphi$ around a top-hat perturbation is derived in the Appendix. The key result is that in the interior of the top hat, $\nabla^{2} \varphi$ is constant like $\nabla^{2} \Psi_{N}$. Hence, a pure top hat will stay top hat, so that Eq. (13) can be considered as an ordinary differential equation involving a spatially constant $\delta$. Note that as shown in Appendix A 7, this is unique to a top hat and will be violated as soon as more general spherically symmetric profiles are considered. Moreover the implied scaling with the local matter density is not true for the exterior of the top hat (cf. [29]). We discuss this issue further in the Appendix.

While $\nabla^{2} \varphi$ is spatially constant for $r \leq R$, it has a nontrivial dependence on $\delta \rho$ (see the Appendix). This dependence can be cast in terms of an effective gravitational constant:

$$
\begin{gathered}
\nabla^{2} \varphi=8 \pi \Delta G_{\mathrm{DGP}}\left(R / R_{*}\right) \delta \rho, \\
\Delta G_{\mathrm{DGP}}(x)=\frac{2}{3 \beta} \frac{\sqrt{1+x^{-3}}-1}{x^{-3}} G,
\end{gathered}
$$

where the Vainshtein radius 


$$
R_{*}=\left(\frac{16 G \delta M r_{c}^{2}}{9 \beta^{2}}\right)^{1 / 3} .
$$

If $R \gg R_{*}, \Delta G_{\mathrm{DGP}}=G /(3 \beta)$ and $\varphi$ is simply proportional to the Newtonian potential, $\Psi_{N}(r)=G \delta M / r$. We call this the linearized limit as it applies to the $\delta M \ll M$ limit.

In the opposite limit relevant for $\delta \rho / \bar{\rho} \gg 1, \Delta G_{\mathrm{DGP}} \propto$ $\left(R / R_{*}\right)^{3 / 2} \ll 1$. This is the Vainshtein limit and here $\varphi \sim$ $G \delta M / R_{*}$ is to leading order constant throughout the perturbation (Appendix A 2).

We can use mass conservation

$$
M=\frac{4 \pi}{3} R^{3} \bar{\rho}(1+\delta)
$$

to rewrite the top-hat equation of motion in Eq. (13) as

$$
\begin{aligned}
\frac{\ddot{R}}{R} & =H^{2}+\dot{H}-\frac{1}{3} \nabla^{2} \Psi \\
& =-\frac{4 \pi G}{3}\left[\bar{\rho}+\left(1+3 w_{\mathrm{eff}}\right) \rho_{\mathrm{eff}}\right]-\frac{4 \pi G_{\mathrm{DGP}}\left(R / R_{*}\right)}{3} \delta \rho,
\end{aligned}
$$

where $G_{\text {DGP }} \equiv G+\Delta G_{\text {DGP }}$. In the second line, the effective equation of state is defined to be $-3\left(1+w_{\text {eff }}\right) \equiv$ $d \ln \rho_{\text {eff }} / d \ln a$. These terms account for the effect of the background expansion.

The terms involving the expansion history can be seen as coming from an effective potential obtained by expanding the Friedmann-Robertson-Walker metric around the center of the perturbation $[19,21]$ :

$$
\Psi_{\mathrm{eff}}=-\frac{1}{2}\left(\frac{\ddot{a}}{a}\right) r^{2}=\frac{2 \pi G}{3}\left(\bar{\rho}+\left(1+3 w_{\mathrm{eff}}\right) \rho_{\mathrm{eff}}\right) r^{2},
$$

up to a constant that is irrelevant for the dynamics (see Appendix A 7). Note that $\nabla^{2} \Psi_{\text {eff }}$ is spatially constant and so its effect also preserves the top-hat profile.

Equation (18) can then be written in compact form as

$$
\frac{\ddot{R}}{R}=\frac{1}{3}\left(\nabla^{2} \Psi_{\text {eff }}+\nabla^{2} \Psi\right)
$$

Note that the pieces involving the matter density combine as

$$
\nabla^{2}\left(\Psi_{\mathrm{eff}}+\Psi_{N}\right)=4 \pi G\left[\rho+\left(1+3 w_{\mathrm{eff}}\right) \rho_{\mathrm{eff}}\right],
$$

so as to reflect the total matter density $\rho$ inside the top hat as one would expect from Newtonian mechanics (see the Appendix for further discussion).

\section{B. Collapse calculation}

We numerically solve the spherical collapse Eq. (18) following [8]. Specifically, we start at an initial scale factor $a_{i}=10^{-5}$, using $\ln a$ as a time variable, and replacing $R$ with $y$ defined as

$$
y \equiv \frac{R}{R_{i}}-\frac{a}{a_{i}}
$$

where $R_{i}$ is the initial radius of the perturbation. Hence, we start with $y=0$ and $y^{\prime}=-\delta_{i} / 3$ as given by linear theory in the matter-dominated epoch in terms of the initial density fluctuation $\delta_{i}$. Here, we have set $\Delta G_{\mathrm{DGP}}=0$ at $a_{i}$, since the effects of force modifications in DGP are negligible at such an early time.

With these initial conditions, we can solve Eq. (18) as

$$
\begin{aligned}
y^{\prime \prime}= & -\frac{H^{\prime}}{H}+y^{\prime}\left(1+\frac{H^{\prime}}{H}\right) y-\frac{\Omega_{m} H_{0}^{2} a^{-3}}{2 H^{2}(a)} \frac{G_{\mathrm{DGP}}\left(R / R_{*}\right)}{G} \\
& \times\left(y+\frac{a}{a_{i}}\right) \delta .
\end{aligned}
$$

The overdensity relative to the background $\delta$ is given by

$$
\delta(y, a)=\left(1+\delta_{i}\right)\left(\frac{a_{i}}{a} y+1\right)^{-3}-1 .
$$

Figure 1 shows the result of solving this equation in the different models (bottom panel). We adjust $\delta_{i}$ so that collapse, where $R=0$ or $y=-a / a_{i}$, occurs at $a=1$. Turnaround, where $d R / d \ln a=0$ or $y^{\prime}=-a / a_{i}$, occurs at $a=0.54-0.56$ for these models.

Figure 1 also shows the evolution of the gravitational force strength $G_{\mathrm{DGP}} / G$. Since collapse is defined by $\delta \rightarrow$ $\infty$ at $a \rightarrow a_{0}$, the perturbation eventually becomes much smaller than its Vainshtein radius in all models, so that $G_{\text {DGP }} \rightarrow G$. Thus, the evolution of forces between turn-

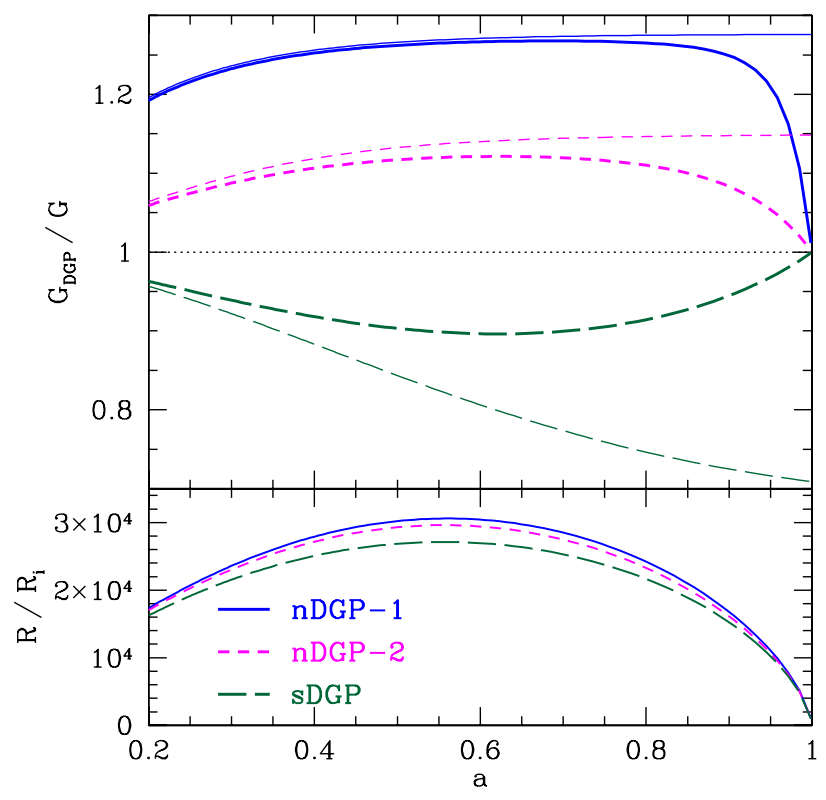

FIG. 1 (color online). Evolution of gravitational force $G_{\mathrm{DGP}} / G$ as a function of $a$ for perturbations collapsing at $a_{0}=1$ in the different DGP models (top panel). In each case, the thin lines show the linearized force modification Eq. (26) whereas the thick lines show the nonlinear case. We also show the evolution of the scaled radius $R / R_{i}$ of the perturbation in the bottom panel. 
around and collapse is significant. This evolution raises the issue of the conservation of total energy of the perturbation during collapse. We will return to this question in the Appendix.

We then extrapolate the initial overdensity $\delta_{i}$ to $a_{0}=1$ using the linear growth equation, obtained from linearizing Eq. (13):

$$
\ddot{\delta}+2 H \dot{\delta}=4 \pi G_{\operatorname{lin}} \bar{\rho} \delta,
$$

where

$$
G_{\mathrm{lin}}(a)=\left[1+\frac{1}{3 \beta(a)}\right] G
$$

is the linearized value of $G_{\mathrm{DGP}}$ for $R / R_{*} \gg 1$ in Eq. (23). The resulting overdensity is the linearly extrapolated collapse overdensity, which we call $\delta_{c}$.

To expose the impact of the Vainshtein mechanism, we will also consider linearized DGP collapse (note that this it not linearized collapse) as the limit of no nonlinear $\varphi$ interactions. In this case, we replace $G_{\text {DGP }}$ with $G_{\text {lin }}$ in Eq. (23). Note that the Vainshtein mechanism is strongest for a spherically symmetric collapse and absent for a planar collapse and so these two cases should encompass the range of possibilities in the cosmological context [9,30].

For a top hat, the Vainshtein radius in units of $R$ is given by $R / R_{*}=(\epsilon \delta)^{-1 / 3}$, where $\epsilon$ is defined as

$$
\epsilon=\frac{8}{9 \beta^{2}(a)}\left(H_{0} r_{c}\right)^{2} \Omega_{m} a^{-3},
$$

such that $1 / \epsilon$ represents the density threshold at which the top-hat perturbation crosses into its own Vainshtein radius.

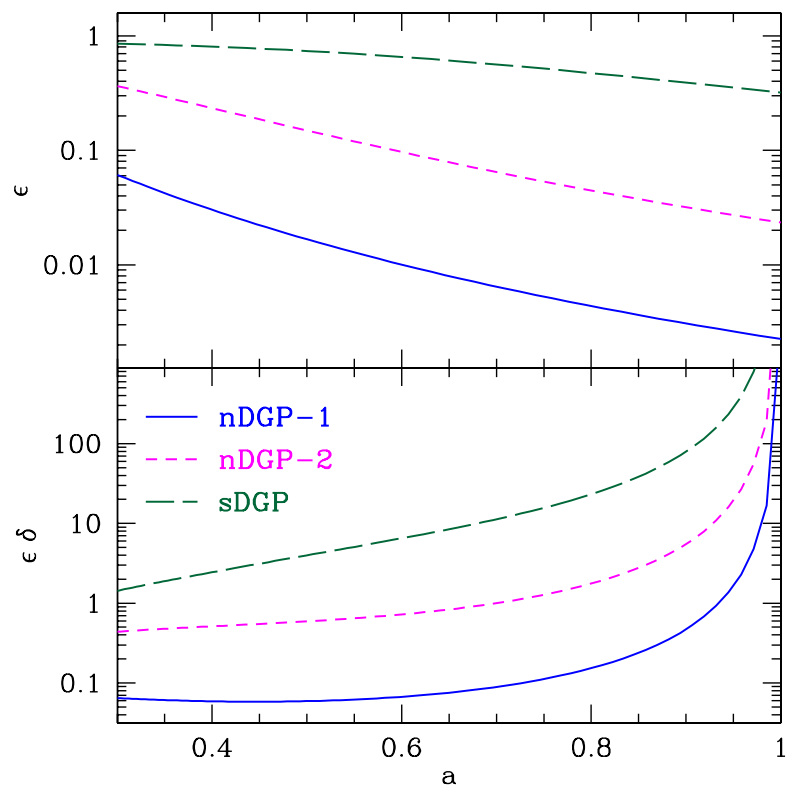

FIG. 2 (color online). Top panel: The nonlinearity parameter $\epsilon$. The Vainshtein mechanism operates once $\epsilon \delta \gtrsim 1$. Bottom panel: $\epsilon \delta$ for a top-hat perturbation that collapses at $a_{0}=1$ in each model.
Figure 2 shows $\epsilon$ and $\epsilon \delta$ as a function of $a$ for a range between turnaround $(a \sim 0.5)$ and collapse $(a=1)$ for the simulated models of Table I. In the nDGP models, this threshold increases substantially toward the present and the Vainshtein suppression does not saturate until quite late in the collapse. In fact in the nDGP-1 model, we shall see that the perturbation virializes before the Vainshtein mechanism can operate.

\section{Virial radius and overdensity}

Spherical top-hat collapse formally predicts a collapse to a singularity at $R \rightarrow 0$. In reality, we expect that processes such as violent relaxation will eventually establish virial equilibrium. More specifically, the virial theorem relates the kinetic energy of the body

$$
T=\int d^{3} x \frac{1}{2} \rho v^{2}=\frac{3}{10} M \dot{R}^{2},
$$

where the last equality holds for a top hat, to the trace of the potential energy tensor

$$
2 T+W=0,
$$

with the definition

$$
W \equiv-\int d^{3} x \rho_{m}(\mathbf{x}) \mathbf{x} \cdot \nabla \Psi .
$$

Note that $W$ depends explicitly on forces only and some care must be taken in relating it to the potential or binding energy of the perturbation for contributions from the branebending mode $\varphi$ (see Appendix A 3). We show there that $W$ can be broken up as a sum of three contributions to the dynamics,

$$
\begin{aligned}
W= & -\frac{3}{5} \frac{G M^{2}}{R}-\frac{4 \pi G}{5}\left(1+3 w_{\mathrm{eff}}\right) \rho_{\mathrm{eff}} M R^{2} \\
& -\frac{3}{5} \frac{\Delta G_{\mathrm{DGP}} M \delta M}{R} .
\end{aligned}
$$

We determine the virial radius $R_{\text {vir }}$ of the perturbation as the radius during collapse (after turnaround) at which Eq. (29) is satisfied. In the literature, conservation of total energy is often used to set this condition. One can easily show that if energy conservation holds strictly, our determination of $R_{\mathrm{vir}}$ agrees with the usual definition. However, in the presence of an evolving $\rho_{\text {eff }}$ and $\Delta G_{\text {DGP }}$, energy is no longer strictly conserved over a Hubble time. Note that this problem occurs in dark energy models as well but is usually ignored under the assumption that $w_{\text {eff }} \approx-1$. However, this procedure is not justified when considering modifications due to a finite $1+w_{\text {eff }}$. Differences between our definition of $R_{\text {vir }}$ and the one relying on energy conservation are of the same order as the differences induced by $1+w_{\text {eff }}$. We discuss these issues further in Appendices A 5 and A 6.

Once $R_{\mathrm{vir}}$ is calculated, we have the density of the perturbation at this radius, $\rho_{\text {vir }}=\bar{\rho}\left(a_{\mathrm{vir}}\right)\left[1+\delta\left(R_{\mathrm{vir}}\right)\right]$, 
TABLE II. Spherical collapse parameters for the cosmologies defined in Table I for collapse at $a_{0}=1$.

\begin{tabular}{ccccc}
\hline \hline & Collapse type/Model: & sDGP & nDGP-1 & nDGP-2 \\
\hline$\delta_{c}$ & GR & 1.662 & 1.674 & 1.674 \\
& DGP & 1.627 & 1.687 & 1.688 \\
& DGP lin. & 1.676 & 1.678 & 1.672 \\
$\Delta_{\text {vir }}$ & GR & 399.9 & 372.3 & 372.3 \\
& DGP & 467.1 & 300.4 & 322.8 \\
& DGP lin. & 436.4 & 311.7 & 339.1 \\
\hline \hline
\end{tabular}

where $a_{\mathrm{vir}}$ is the scale factor at which the perturbation reaches $R_{\text {vir }}$ during collapse. For collapse at $a_{0}=1, a_{\text {vir }} \approx$ $0.91-0.93$ for the simulated models. One then assumes that the perturbation maintains this "virial density" while the background continues to decrease. The final virial density with respect to the background, $\Delta_{\text {vir }}$ at $a_{0}$ is then given by referring this density to the background matter density at $a_{0}$ :

$$
\Delta_{\mathrm{vir}}=\left[1+\delta\left(R_{\mathrm{vir}}\right)\right]\left(\frac{a_{0}}{a_{\mathrm{vir}}}\right)^{3} .
$$

Table II shows the resulting spherical collapse parameters, $\delta_{c}$ and $\Delta_{\text {vir }}$, for the different models and gravitational modifications: unmodified (GR collapse), valid for GR with smooth dark energy; the expression Eq. (15) (DGP collapse); and Eq. (26) (linearized DGP collapse).

\section{HALO MODEL PREDICTIONS}

We now briefly describe how we move from spherical collapse predictions of the linear collapse threshold $\delta_{c}$ and the virial overdensity $\Delta_{\text {vir }}$ summarized in Table II to predictions of the halo mass function, bias, and nonlinear power spectrum. For further details, see [8].

In the Press-Schechter approach, one assumes that all regions with $\delta>\delta_{c}$ in the linearly extrapolated initial density field collapse to form bound structures (halos). The fraction of mass within halos at a given mass is then determined by the variance of the linear density field smoothed at that scale. Here, we adopt the Sheth-Tormen (ST) prescription [31] for the halo mass function predictions, which enables a direct use of our spherical collapse results. Also, we previously found a good match to the ST mass function and bias in our $\Lambda \mathrm{CDM}$ simulations [8].

The ST description for the comoving number density of halos per logarithmic interval in the virial mass $M_{\mathrm{vir}}$ is given by

$$
n_{\ln M_{\text {vir }}} \equiv \frac{d n}{d \ln M_{\mathrm{vir}}}=\frac{\bar{\rho}}{M_{\mathrm{vir}}} f(\nu) \frac{d \nu}{d \ln M_{\mathrm{vir}}},
$$

where the peak threshold $\nu=\delta_{c} / \sigma\left(M_{\text {vir }}\right)$ and

$$
\nu f(\nu)=A \sqrt{\frac{2}{\pi} a \nu^{2}}\left[1+\left(a \nu^{2}\right)^{-p}\right] \exp \left[-a \nu^{2} / 2\right]
$$

Here, the virial mass is defined as the mass enclosed at the virial radius $R_{\mathrm{vir}} . \sigma(M)$ is the variance of the linear density field convolved with a top hat of radius $R$ that encloses $M=4 \pi R^{3} \bar{\rho} / 3$ at the background density

$$
\sigma^{2}(R)=\int \frac{d^{3} k}{(2 \pi)^{3}}|\tilde{W}(k R)|^{2} P_{\mathrm{L}}(k),
$$

where $P_{\mathrm{L}}(k)$ is the linear power spectrum and $\tilde{W}$ is the Fourier transform of the top-hat window. The normalization constant $A$ in Eq. (34) is chosen such that $\int d \nu f(\nu)=$ 1. We adopt the standard parameter values of $p=0.3$ and $a=0.75$ throughout.

The linear bias corresponding to the ST mass function, obtained in the peak-background split, is given by [31]

$$
\begin{aligned}
b_{\text {lin }}\left(M_{\mathrm{vir}}\right) & \equiv b\left(k=0, M_{\mathrm{vir}}\right) \\
& =1+\frac{a \nu^{2}-1}{\delta_{c}}+\frac{2 p}{\delta_{c}\left[1+\left(a \nu^{2}\right)^{p}\right]} .
\end{aligned}
$$

By assuming a specific form of halo density profiles, we can rescale mass definitions from the virial mass $M_{\text {vir }}$ to $M_{200}$, the mass definition used in the simulation measurements, as outlined in [32] (again, all overdensities are referred to the background matter density). We use this approach to compare the scaling relation predictions to the simulations in Sec. V. For the halo profiles, we take an Navarro-Frenk-White form [33],

$$
\rho_{\mathrm{NFW}}(r)=\frac{\rho_{s}}{r / r_{s}\left(1+r / r_{s}\right)^{2}},
$$

where $r_{s}$ is the scale radius of the halo and the normalization $\rho_{s}$ is given by the virial mass $M_{\mathrm{vir}}$. We parametrize $r_{s}$ via the concentration $c_{\text {vir }} \equiv R_{\text {vir }} / r_{s}$ given by [34]

$$
c_{\mathrm{vir}}\left(M_{\mathrm{vir}}, z=0\right)=9\left(\frac{M_{\mathrm{vir}}}{M_{*}}\right)^{-0.13},
$$

where $M_{*}$ is defined via $\sigma\left(M_{*}\right)=\delta_{c}$. Since generally $R_{\text {vir }}$, $R_{200} \gg r_{s}$, the precise form of the concentration relation has a negligible impact on the mass rescaling. In the following, when no specific overdensity is given, we implicitly take $M=M_{200}$, e.g.

$$
n_{\ln M} \equiv \frac{d n}{d \ln M_{200}}=n_{\ln M_{\text {vir }}} \frac{d \ln M_{\mathrm{vir}}}{d \ln M_{200}} .
$$

We also consider the nonlinear matter power spectrum calculated in the halo model approach (see [17] for a review). Since all matter is assumed to be within bound halos, the matter power spectrum can be decomposed into 1-halo and 2-halo terms,

$$
P_{\mathrm{mm}}(k)=I^{2}(k) P_{\mathrm{L}}(k)+P^{1 \mathrm{~h}}(k),
$$

where 


$$
\begin{aligned}
P^{\operatorname{lh}}(k) & =\int d \ln M_{\mathrm{vir}} n_{\ln M_{\mathrm{vir}}} \frac{M_{\mathrm{vir}}^{2}}{\bar{\rho}_{\mathrm{m}}^{2}}\left|y\left(k, M_{\mathrm{vir}}\right)\right|^{2}, \\
I(k) & =\int d \ln M_{\mathrm{vir}} n_{\ln M_{\mathrm{vir}}} \frac{M_{\mathrm{vir}}}{\bar{\rho}_{\mathrm{m}}} y\left(k, M_{\mathrm{vir}}\right) b_{\mathrm{lin}}\left(M_{\mathrm{vir}}\right) .
\end{aligned}
$$

Here, $y(k, M)$ is the Fourier transform of an NavarroFrenk-White density profile truncated at $R_{\text {vir }}$, and normalized so that $y(k, M) \rightarrow 1$ as $k \rightarrow 0$. Note that with the ST mass function and bias, $\lim _{k \rightarrow 0} I(k)=1$.

\section{RESULTS}

We compare our spherical collapse and halo model predictions with the results of $N$-body simulations presented in $[9,26]$ of the sDGP and nDGP + DE models (see Sec. II, Table I). In addition to the full simulations which solve the nonlinear $\varphi$ Eq. (9), simulations using the linearized $\varphi$ equation have been performed through Eq. (26).

We always compare observables measured in the DGP simulations with those of GR simulations with the same initial conditions and expansion history. In this way, cosmic variance as well as systematic issues cancel out to a large extent.

\section{A. Halo mass function}

Figure 3 shows the deviation of the halo mass function from QCDM measured in the sDGP and linearized sDGP

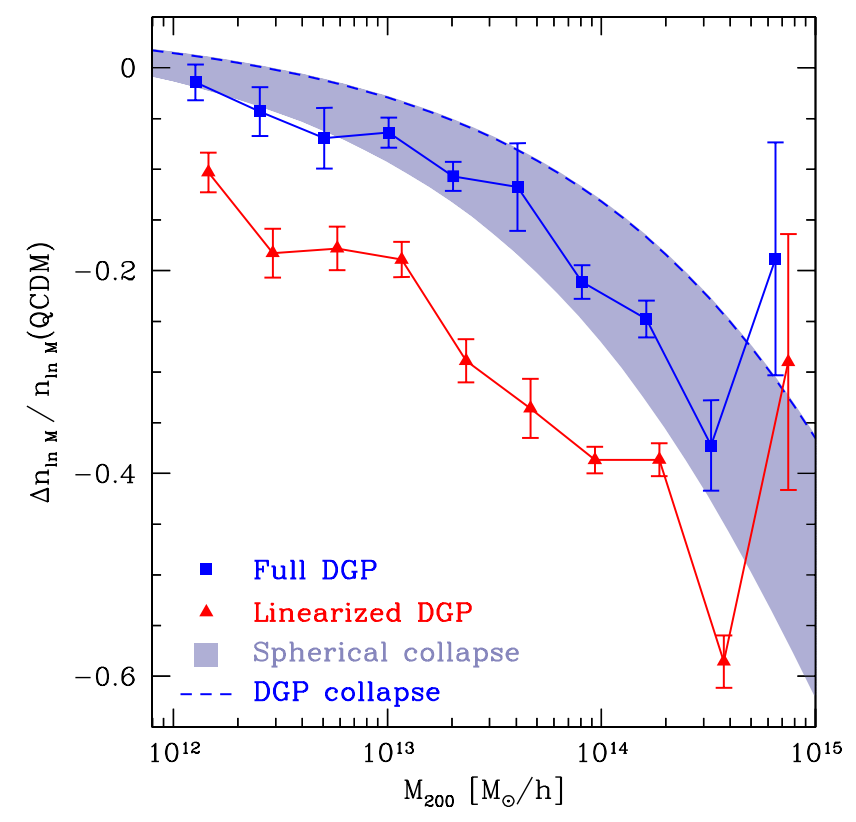

FIG. 3 (color online). Deviation in the halo mass function at $z=0$ of sDGP from a dark energy model with the same expansion history (QCDM). The points show measurements in the full and linearized DGP simulations, the band shows the Sheth-Tormen + spherical collapse prediction range between full DGP collapse (blue dashed line) and linearized DGP collapse.

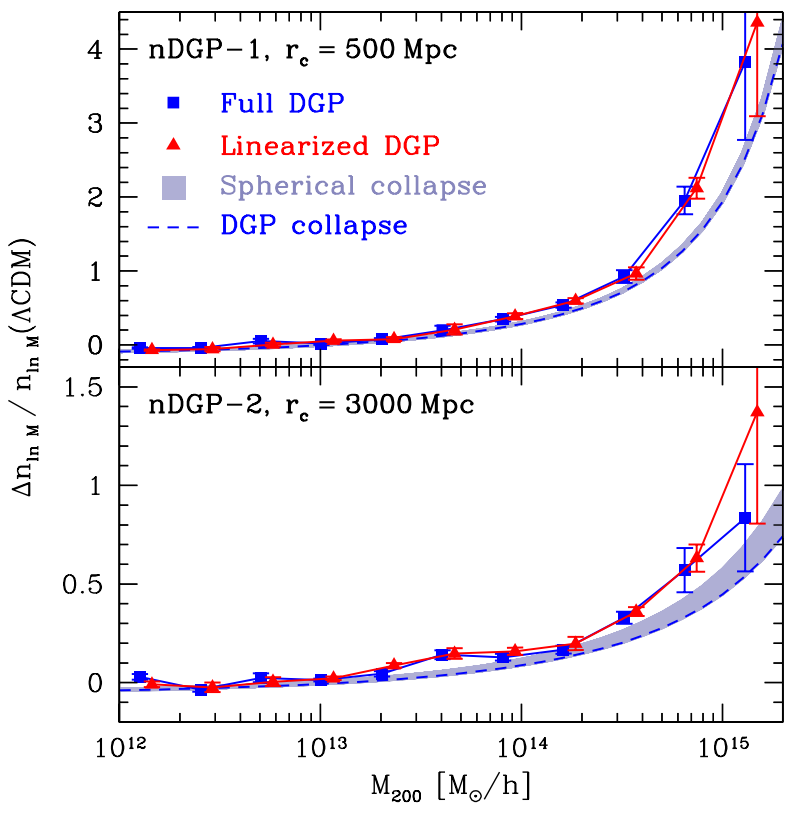

FIG. 4 (color online). Same as Fig. 3, for the two normal branch DGP + dark energy models nDGP-1 (top) and nDGP-2 (bottom) relative to $\Lambda \mathrm{CDM}$. The linearized DGP simulation results have been displaced horizontally for clarity.

simulations, and the spherical collapse predictions. Figure 4 shows the corresponding results for the nDGP + DE models. The spherical collapse predictions work well in both cases and, in particular, match the shape of the deviations and the relative impact of the Vainshtein mechanism. In both cases, they somewhat underestimate the size of the deviations at a fixed mass, corresponding roughly to a shift in $\lg M_{200}$ of $\sim 0.3-0.5$.

In the nDGP models, force modifications are stronger (see Fig. 2), leading to larger deviations in the mass function from the corresponding GR model with the same expansion history. In particular, the abundance of massive halos $M_{200} \gtrsim 10^{14} M_{\odot} / h$ is significantly enhanced. This behavior is due to the exponential sensitivity of the mass function to $\nu=\delta_{c} / \sigma(M)$ at the high-mass end, and is similar to what was seen in the large-field $f(R)$ models in [8].

Furthermore, the density threshold $\epsilon^{-1}$ for the onset of the Vainshtein mechanism is higher in the nDGP models than the sDGP model, so that the mass function is less affected by the Vainshtein mechanism in nDGP. This is borne out by both simulations and spherical collapse predictions: the relative spread in the predictions between the DGP and linearized DGP case shrinks considerably when going from large to small $r_{c}$, i.e. from sDGP to nDGP-2 to nDGP-1.

Finally, since the full spherical collapse predictions always slightly underestimate the deviations, they can be used to place conservative limits on DGP braneworld scenarios from measurements of the halo mass function e.g. from massive clusters [35-37]. Alternatively, the pre- 
dictions can be recalibrated based on simulations by introducing a constant shift in $\lg M_{200} \sim 0.3-0.5$.

\section{B. Halo bias}

This section presents new results on the clustering of halos in the simulations of $[9,26]$. We extract the linear halo bias $b_{\text {lin }}(M)$ from our simulations as described in [8]. For halos of a given logarithmic mass range in a box of size $L_{\text {box }}$, we first obtain the halo bias $b(k, M)$ by dividing the halo mass cross spectrum $P_{\mathrm{hm}}(k)$ by the matter power spectrum for each simulation run. ${ }^{2}$ In order to remove trends from the nonlinearity of the bias, we then fit a linear relation to $b(k, M)=b_{\text {lin }}(M)+a(M) k$ between the minimum $k$ (the fundamental mode of the box) and $\sim 15 k_{\min }$, where $b(k, M)$ is the combined measurement from all boxes. The same fitting procedure is applied to the runby-run ratio of $b_{\mathrm{DGP}}(k, M) / b_{\mathrm{GR}}(k, M)$.

We then bootstrap over many realizations of the set of simulations, performing the fit for every realization. We use the average of the fit parameter $b_{\text {lin }}(M)$ as estimate of the linear bias, and its spread as an estimate of the error. Note that in case of the nDGP simulations, we only have 3 runs per box size, so that the error estimate itself has a large uncertainty.

We show the linear bias $b_{\text {lin }}(M)$ as a function of halo mass for the QCDM and $\Lambda$ CDM simulations themselves in Fig. 5. As the halo mass function deviates significantly from a pure power law, especially at the high-mass end, we plot the bias measurements at the position of the measured average $\lg M_{200}$ of the halos. The Sheth-Tormen prediction of Eq. (36), using the parameters from Table II and rescaled from $M_{\text {vir }}$ to $M_{200}$ in each case, matches the simulations well for masses up to $10^{14} M_{\odot} / h$. At higher masses, it overpredicts the bias in the simulations, though the deviation is a the level of $1-2 \sigma$. Note that halos are more biased at a given mass in QCDM than in $\Lambda \mathrm{CDM}$, due to the reduced growth and smaller power spectrum amplitude in this model.

This trend continues in the DGP simulations: in sDGP, gravity is further weakened by the repulsive brane-bending mode, so that the linear halo bias at fixed mass is increased by $\sim 10 \%$ compared to QCDM (Fig. 6). There is a hint that halos are slightly higher biased in the linearized simulations compared to the full simulations, though both are consistent given the error bars. The spherical collapse prediction matches the simulation results over the whole mass range. The full DGP collapse (blue dashed line) marks the lower edge of the shaded band, while the linearized DGP collapse corresponds to the upper edge, in accordance with expectations. The spread of the predic-

\footnotetext{
${ }^{2}$ Note that the definition of bias adopted will differ from alternate choices such as $\left(P_{\mathrm{hh}} / P_{\mathrm{mm}}\right)^{1 / 2}$ or $P_{\mathrm{hh}} / P_{\mathrm{hm}}$ in the nonlinear regime where the correlation coefficient between halos and matter can differ from unity.
}

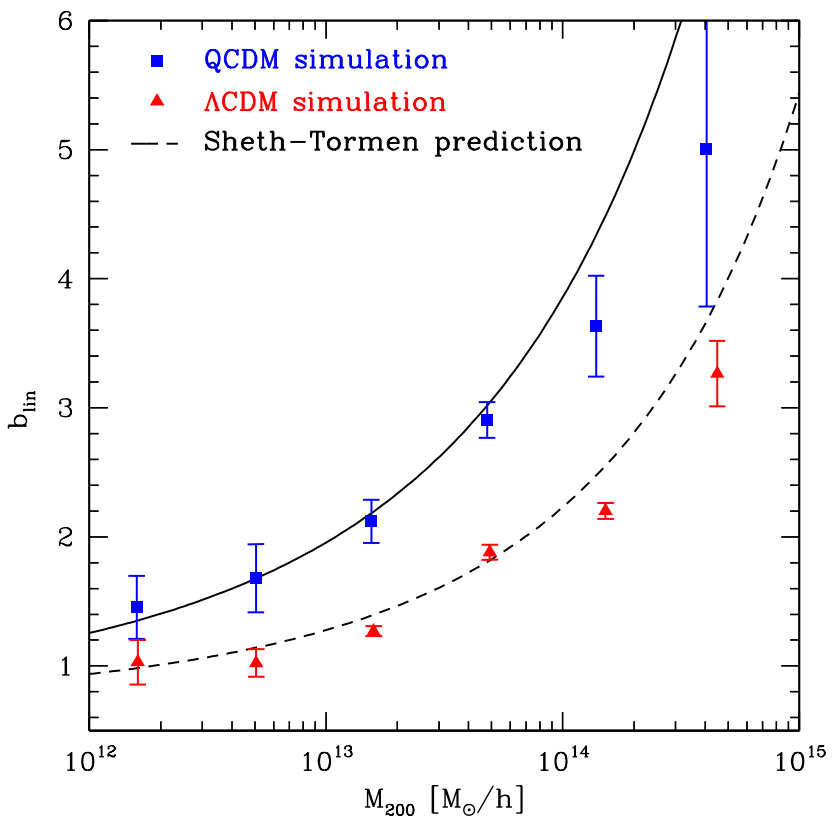

FIG. 5 (color online). Linear bias $b_{\text {lin }}$ of dark matter halos measured in the $\Lambda \mathrm{CDM}$ and QCDM simulations at $z=0$ (see Sec. V B), and the prediction of the Sheth-Tormen prescription.

tions is similar in magnitude to the tentative differences between linearized and full simulations.

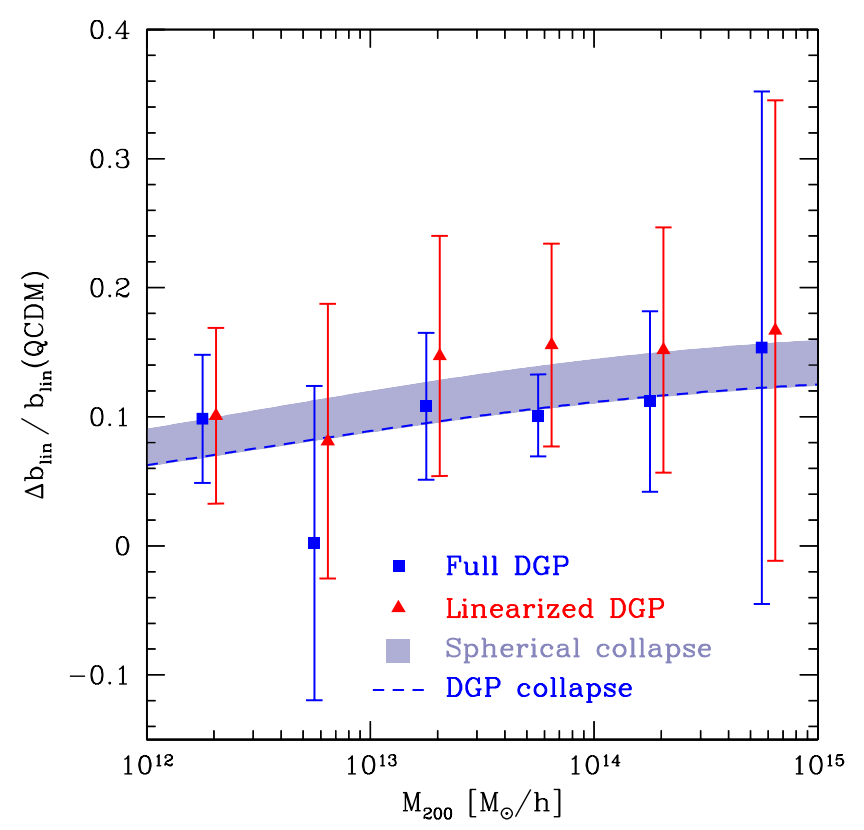

FIG. 6 (color online). Relative deviation of the linear bias $\Delta b_{\text {lin }} / b_{\text {lin }}$ in the full and linearized sDGP simulations from that of the QCDM simulations as a function of halo mass at $z=$ 0 . The linearized simulation points have been displaced horizontally for clarity. The band shows the Sheth-Tormen + spherical collapse prediction range between full DGP collapse (blue dashed line) and linearized DGP collapse. 


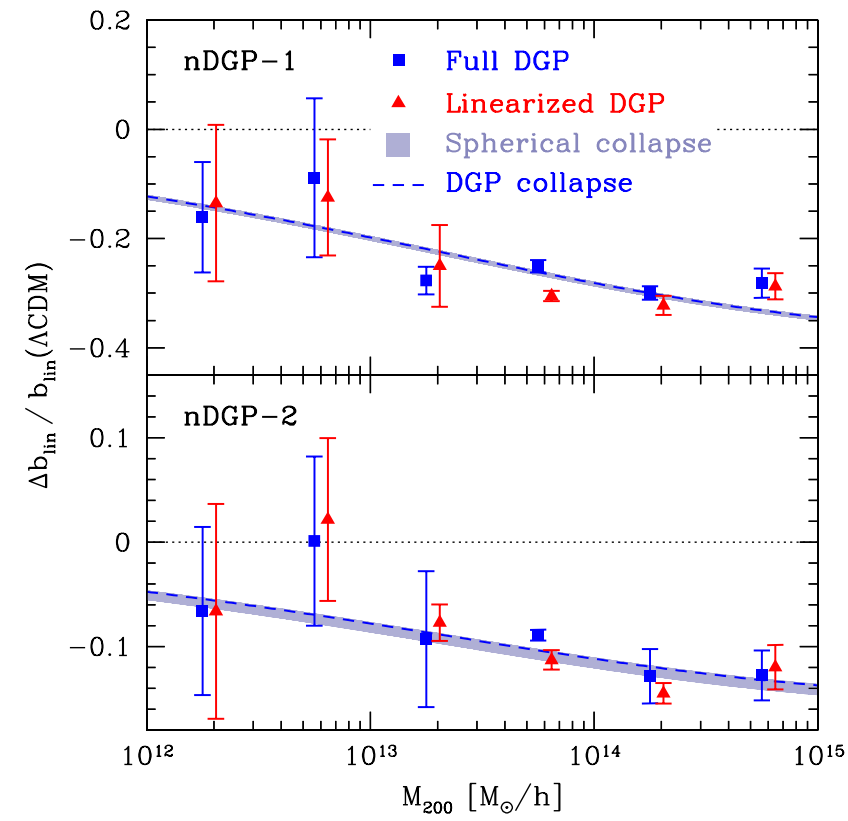

FIG. 7 (color online). Same as Fig. 6, for the two normal branch DGP + dark energy models nDGP-1 (top) and nDGP-2 (bottom), relative to $\Lambda \mathrm{CDM}$.

For the nDGP models, the opposite trend in the bias is seen (Fig. 7): halos are less biased at a given mass. While the full and linearized simulation results are consistent in the nDGP models, showing no sign of the Vainshtein mechanism affecting the linear halo bias, they both follow the spherical collapse prediction very well. This is in accordance with the good description of the mass function results in Sec. VA. Also, given the small spread in the spherical collapse predictions, we do not expect to see any differences between full and linearized simulations for the nDGP models.

\section{Nonlinear matter power spectrum}

We can now assemble the ingredients of the halo model: the mass function, bias, and profile of halos, to predict the nonlinear matter power spectrum following Sec. IV. We assume that the inner parts of the density profiles of halos are not affected by DGP, as indicated by simulations [26]. More precisely, one can assume that for a halo of given mass $M_{200}$, the scale radius $r_{s}$ [Eq. (37)] is the same in DGP as in GR. Adopting Eq. (38) for the concentration $c_{\mathrm{vir}}=R_{\mathrm{vir}} / r_{s}$ in GR, we have for the concentration relation in DGP:

$$
c_{\mathrm{vir}, \mathrm{DGP}}(M)=\frac{\left.R_{\Delta}(M)\right|_{\Delta_{\mathrm{vir}}(\mathrm{DGP})}}{\left.R_{\Delta}(M)\right|_{\Delta_{\mathrm{vir}}(\mathrm{GR})}} c_{\mathrm{vir}}\left(M_{\mathrm{vir}, \mathrm{GR}}\right),
$$

where $R_{\Delta}(M)=(3 M /(4 \pi \Delta \bar{\rho}))^{1 / 3}$, and $M_{\text {vir,GR }}$ is the virial mass in GR that corresponds to a virial mass of $M$ in DGP. We find that for all DGP models considered here, the concentration $c_{\mathrm{vir}, \mathrm{DGP}}$ defined by Eq. (42) is within 3\% of

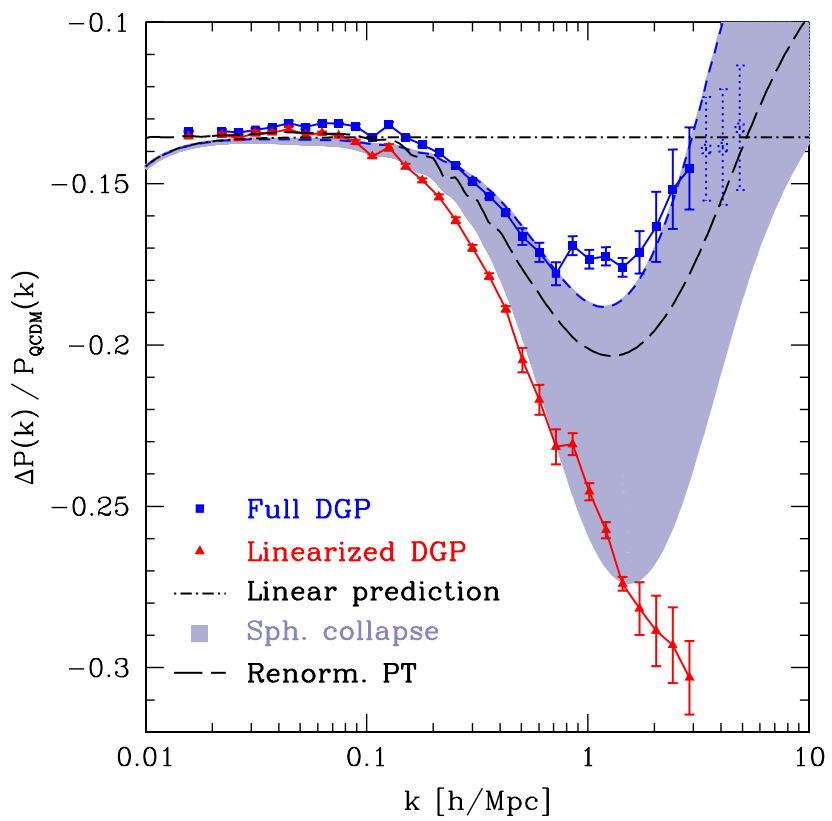

FIG. 8 (color online). Deviation in the matter power of the sDGP model from QCDM at $z=0$. The points show measurements in the full and linearized DGP simulations, while the band shows the halo model prediction based on spherical collapse and the Sheth-Tormen prescription [between DGP (blue dashed line) and linearized DGP collapse]. The long-dashed line shows the renormalized perturbation theory prediction from [30].

the standard relation $c_{\mathrm{vir}}\left(M_{\mathrm{vir}, \mathrm{DGP}}\right)$, which has a negligible effect on the power spectrum on the scales probed by the simulations. Hence, we leave the concentration relation Eq. (38) unchanged in our power spectrum predictions.

Figure 8 shows that the sDGP simulation results are matched very well: the DGP collapse prediction (blue dashed line) is close the to full simulation results, while the linearized DGP collapse is close to the linearized simulations. The renormalized perturbation theory prediction of [30], which uses a completely different approach to take into account the nonlinear interactions of the branebending mode, is also quite close to our DGP collapse calculation.

The match to the power spectrum in the nDGP simulations (Fig. 9) is somewhat worse, showing discrepancies of $\sim 30 \%$ for nDGP-1 and $\sim 10 \%$ for nDGP-2. In particular, discrepancies can be seen in the quasilinear to nonlinear transition, $k \sim 0.1-1 h / \mathrm{Mpc}$ for $\mathrm{nDGP}-1$. The simplified 1halo/2-halo split in the halo model breaks down on these scales. We also show the predicted nonlinear power spectra using halofit [38] in combination with the linear DGP power spectra at $z=0$. Note that for $k \gtrsim 0.01 h / \mathrm{Mpc}$, the linear DGP power spectrum is identical to that of a $\Lambda$ CDM model with higher linear normalization. While the match to the simulations is better than our spherical collapse predictions at $k \leqq 0.1 \mathrm{~h} / \mathrm{Mpc}$, the deviations grow towards smaller scales so that halofit is a worse fit to the simulations than the spherical collapse model for $k \gtrsim$ 


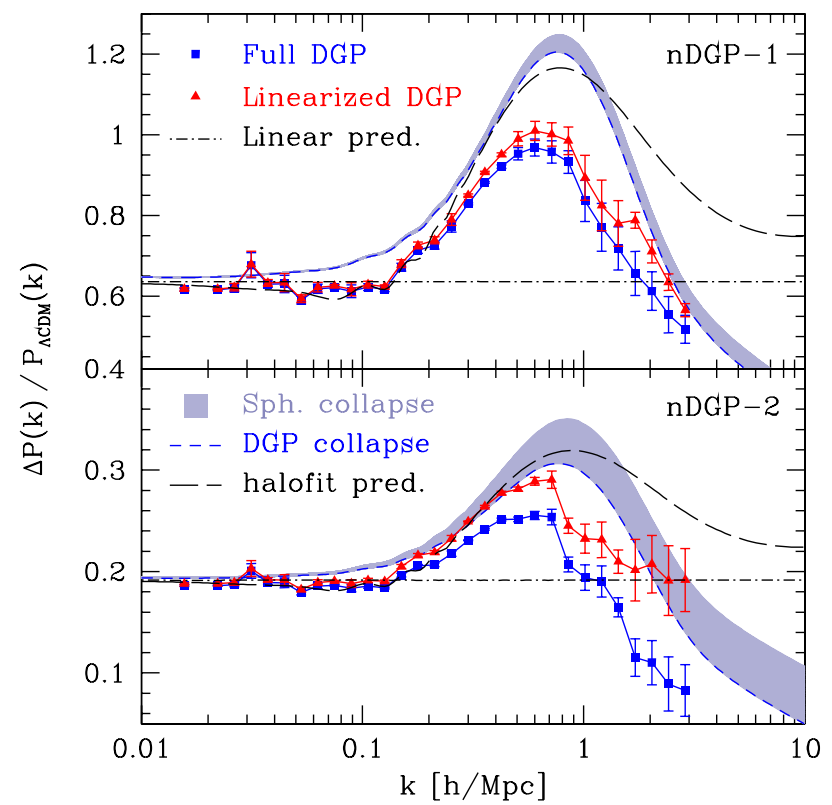

FIG. 9 (color online). Same as Fig. 8, for the two normal branch DGP + dark energy models nDGP-1 (top) and nDGP-2 (bottom) relative to $\Lambda \mathrm{CDM}$. The long-dashed lines show the predictions of halofit using the linear DGP power spectrum.

$1 \mathrm{~h} / \mathrm{Mpc}$. Clearly, it is not trivial to model the simulation results for $\Delta P(k) / P(k)$ to better than $\sim 10 \%$.

While the overall magnitude of the power spectrum enhancement in nDGP is not matched, the shape of $\Delta P(k) / P(k)$ is matched quite well. Furthermore, the effect of the Vainshtein mechanism on the power spectrum is predicted accurately, as can be seen by comparing the spread in the spherical collapse predictions to the difference between full and linearized simulation results. These findings indicate that it should be possible to rescale linearized DGP simulation results with the relative Vainshtein suppression calculated from spherical collapse.

\section{CONCLUSIONS}

By studying the collapse of a spherical perturbation under DGP braneworld gravity, we have shown how simulation results on the mass function, halo bias and power spectrum can be understood semianalytically. In DGP gravity, force modifications are carried by the scalar brane-bending mode. The global properties of the response of the brane-bending mode to matter control how the Vainshtein mechanism modifies force and energy conditions during collapse. These conditions are important for the calculation of virial equilibrium (see the Appendix for detailed discussions of these results).

In particular, the presence of evolving modifications to the gravitational force either through the brane-bending mode or through the background expansion violate conservation of Newtonian total energy for traditional definitions of the potential energy contribution. This violation applies to smooth dark energy models with $w \neq-1$ as well. We introduce a new, general technique for defining the virial radius which does not rely on strict energy conservation.

Under the halo model, these spherical collapse predictions give rise to predictions for the mass function, halo bias, and power spectrum. We have shown that these predictions are in good qualitative agreement with DGP $N$-body simulations on both the self-accelerating and normal branch. In particular, the use of spherical collapse for the mass function always provides slightly conservative limits on mass function deviations when compared with the simulations. Hence, the semianalytic techniques introduced here can be used as a practical tool for extending simulation results for the purpose of studying parameter constraints on braneworld models from observations of the mass function.

While the absolute power spectrum agreement is not quite as good, these techniques can still be useful in combination with linearized DGP simulations. Since our spherical collapse predictions appear to capture accurately the impact of the Vainshtein mechanism on the power spectrum, they could provide an effective way of taking nonlinear interactions into account in results obtained from linearized DGP simulations. Such simulations are very easy to implement and an order of magnitude cheaper computationally than the full DGP simulations. Likewise, they are more readily extendable to higher resolution and can be used to cover a wider range in parameter space.

\section{ACKNOWLEDGMENTS}

The simulations used in this work have been performed on the Joint Fermilab-KICP Supercomputing Cluster, supported by grants from Fermilab, Kavli Institute for Cosmological Physics, and the University of Chicago. F.S. acknowledges support from the Gordon and Betty Moore Foundation at Caltech. This work was supported by the Kavli Institute for Cosmological Physics at the University of Chicago through NSF Grants No. PHY0114422 and No. PHY-0551142. W. H. was additionally supported by DOE Contract No. DE-FG02-90ER-40560 and the Lucile and David Packard Foundation. M. L. was supported by an NSF-PIRE grant.

\section{APPENDIX A: TOP-HAT OVERDENSITY IN DGP}

In this Appendix, we present in detail the various aspects of top-hat perturbations in DGP used in the main text. We begin by reviewing the techniques introduced in Ref. [6] for the brane-bending field but pay special attention to the matching of the various solutions as well as derive a closed form expression for the global field profile. This global profile is used to study virial equilibrium (Appendix A 3) and potential energy (Appendix A 4). We discuss subtleties due to the relationship between the two and violations of Newtonian energy conservation in Appendix A 5 and their 
impact on defining the virial overdensity in Appendix A 6. Finally, we examine the impact of density compensation in the exterior of the top hat in Appendix A 7 in light of the lack of a Birkhoff theorem in DGP.

\section{Spherical symmetry}

We begin by assuming that the density perturbation $\delta \rho(r)$ is spherically symmetric but otherwise arbitrary. Given the induced spherical symmetry in the field solution, the field Eq. (9) reduces to

$$
\frac{1}{r^{2}} \frac{d}{d r} r^{2} \frac{d \varphi}{d r}+\frac{r_{c}^{2}}{3 \beta}\left[\frac{4}{r} \frac{d^{2} \varphi}{d r^{2}} \frac{d \varphi}{d r}+2\left(\frac{1}{r} \frac{d \varphi}{d r}\right)^{2}\right]=\frac{8 \pi G}{3 \beta} \delta \rho .
$$

Integration of the field Eq. (A1) over $r^{2} d r$ then yields

$$
r^{2} \frac{d \varphi}{d r}+\frac{2}{3 \beta} r_{c}^{2} r\left(\frac{d \varphi}{d r}\right)^{2}=\frac{2}{3 \beta} G m(r),
$$

with the enclosed mass perturbation defined as

$$
m(r) \equiv 4 \pi \int_{0}^{r} r^{\prime 2} \delta \rho\left(r^{\prime}\right) d r^{\prime} .
$$

If $\delta \rho(r)=0$ for $r>R$, the enclosed mass fluctuation $m(r>R)$ is the total mass fluctuation $\delta M$.

Note that the $\nabla_{i} \nabla_{j} \varphi$ term in Eq. (9) is critical in obtaining this solution, since it causes cancellation of the integral terms when integrating by parts, leaving only the boundary terms for the nonlinear piece. The field solution interior to $r$ has no direct effect on the solution at $r$. Like Newtonian dynamics, only the enclosed mass, not the enclosed field, matters. This property is crucial for maintaining the linearity of the field solution at large $r$ in the presence of strong nonlinearity at small $r$. More generally, linearity is a consequence of Eq. (9) satisfying a nonlinear Gauss's law and does not require spherical symmetry (see e.g. [39]).

Since $m \rightarrow \delta M$ at distances beyond which there are no density fluctuations, and assuming that $\varphi(\infty)=0$, we can immediately see that the far exterior solution must be to leading order $\varphi \propto 1 / r$. Given an increasingly small nonlinear term, this requires

$$
\lim _{r \rightarrow \infty} \varphi=-\frac{2}{3 \beta} \frac{G \delta M}{r} .
$$

For the small $r$ solution, the key simplification is that the force modification $d \varphi / d r$ is now an analytic function of the enclosed mass

$$
\frac{d \varphi}{d r}=\frac{3 \beta r_{*}(r)}{4 r_{c}^{2}} g\left(r / r_{*}\right),
$$

where the Vainshtein radius of the enclosed mass is

$$
r_{*}(r)=\left(\frac{16 G m(r) r_{c}^{2}}{9 \beta^{2}}\right)^{1 / 3}
$$

and

$$
g(x)=x\left[\sqrt{1+x^{-3}}-1\right] .
$$

Note that in general $r_{*}$ is a function of $r$ and reflects the enclosed mass, and $r_{*}^{3} / r^{3}$ the average density, not the local density. The latter would be implied by setting

$$
\left(\nabla_{i} \nabla_{j} \varphi\right)^{2}=c\left(\nabla^{2} \varphi\right)^{2}
$$

with $c=$ const in the original field equation. However, doing so would violate the $r \rightarrow \infty$ limit. In particular, the far field limit in this approximation would reveal the presence of a Vainshtein screened mass instead of the true mass perturbation $\delta M$. Small scale nonlinearity in the density field would then no longer average to give the required linear perturbations on large scales [40]. Such an approximation or any that relates the field solution to the local density should not be used in a cosmological context (cf. $[26,29]$ ).

\section{Field profile}

We now assume a top-hat spherical perturbation of radius $R$ with a constant density enhancement $\delta \rho$ [Eq. (11)]. As usual, we neglect any compensating underdensity swept out by the prior evolution of the top hat. Since the force modifications within the top hat only depend on the enclosed mass $m(r)$ and not the exterior, the compensation does not impact the dynamics. It can however influence the field profile $r>R$ and we return to this point in Appendix A 7. For the pure top hat, $m(r \geq$ $R)=\delta M$ and we can solve for the profile $\varphi(r)$ in closed form.

First, let us consider the exterior solution at $r>R$. In the exterior, $m=$ const and there is a single Vainshtein radius $R_{*}=r_{*}(R)$. Defining a new variable $x=r / R_{*}$, we can write Eq. (A5) as

$$
\frac{d \varphi}{d x}=A g(x)
$$

where

$$
A=\frac{3 \beta}{4}\left(\frac{R_{*}}{r_{c}}\right)^{2}=\frac{4}{3 \beta} \frac{G \delta M}{R_{*}},
$$

and we can obtain the full exterior field solution as

$$
\begin{aligned}
\varphi(r) & =-\int_{r / R_{*}}^{\infty} \frac{d \varphi}{d x} d x \\
& =\frac{A x^{2}}{2}\left\{{ }_{2} F_{1}\left[-1 / 2,-2 / 3,1 / 3,-1 / x^{3}\right]-1\right\} .
\end{aligned}
$$

The solution of course recovers Eq. (A4) in the limit of $x \gg 1$,

$$
\lim _{x \gg 1} \varphi=-\frac{A}{2 x}=-\frac{2}{3 \beta} \frac{G \delta M}{r} .
$$

Note that the constant $A$ is $-2 \varphi_{*}$, where $\varphi_{*}$ is the linearized field profile evaluated at the Vainshtein radius. 
In the opposite limit $x \ll 1$,

$$
\lim _{x \ll 1} \varphi=-A\left(C_{0}-2 x^{1 / 2}\right) .
$$

The constant piece,

$$
C_{0} \equiv A^{-1} \int_{0}^{\infty} d x \frac{d \varphi}{d x}=\frac{\Gamma[1 / 3] \Gamma[1 / 6]}{4 \sqrt{\pi}} \approx 2.103,
$$

therefore dominates, and

$$
\lim _{x \ll 1} \varphi \approx-\frac{4 C_{0}}{3 \beta} \frac{G \delta M}{R_{*}}=\text { const. }
$$

However, field gradients are determined solely by the $x^{1 / 2}$ term. Since particle dynamics depend on forces and hence field gradients, the existence of a constant term in addition to the $x^{1 / 2}$ term in the field profile makes an important difference when comparing energy conditions like conservation laws and dynamical considerations like virial equilibrium, as we shall see. This distinction is often neglected in the literature (e.g. $[28,29])$. Figure 10 shows $|\varphi(\delta M ; R)|$ in units of the linearized $(x \gg 1)$ value as a function of $R / R_{*}$. Note the strong suppression of the surface potential and its saturation for $R \ll R_{*}$.

The field interior to the top hat can be similarly solved. Since $r_{*}(r) \propto r$ for a constant density profile, Eq. (A5) implies $d \varphi / d r \propto r$ and hence

$$
\varphi(r)-\varphi(0)=B r^{2}
$$

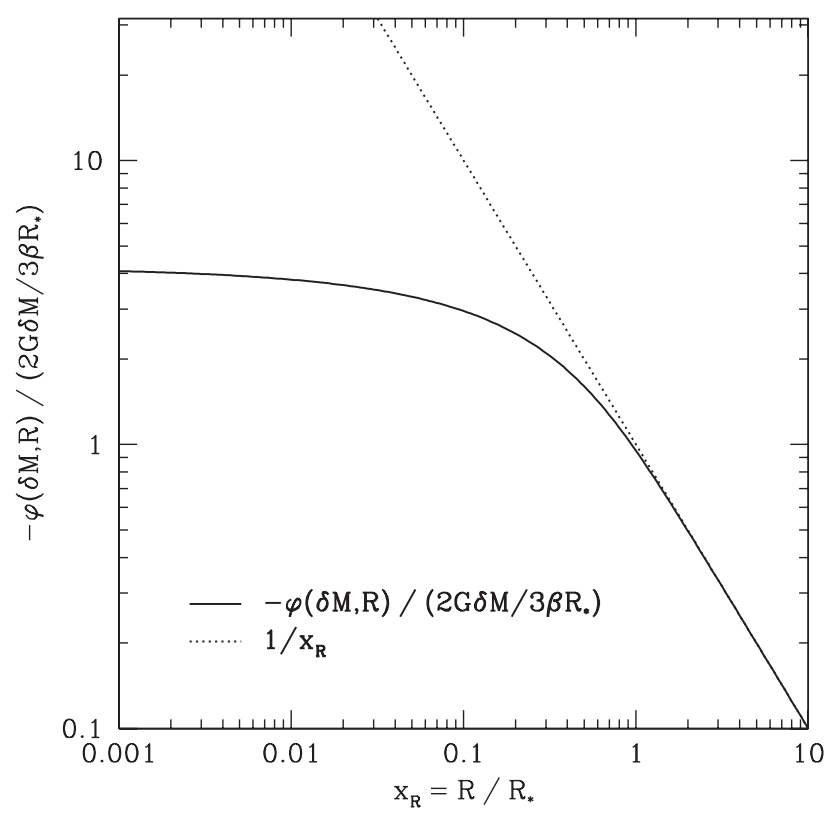

FIG. 10. Surface field profile $|\varphi(\delta M, R)|$ [solid curve, see Eq. (A18)] in units of $(2 / 3 \beta) G \delta M / R_{*}$, the linearized value at $R_{*}$, as function of $x_{R}=R / R_{*}$. Parameters are for the sDGP model $(a=1)$. The dotted line shows the linearized solution, $x_{R}^{-1}$ in these units. In the Vainshtein limit $x_{R} \ll 1, \varphi(\delta M, R)$ approaches a constant. $\varphi(\delta M, R)$ also determines the binding energy $U_{\varphi}$ (Appendix A 4).
We see from substitution into Eq. (A1) that

$$
B=\frac{A}{2 R R_{*}} g\left(x_{R}\right)
$$

solves the field equation. Here, $x_{R}=x(R)=R / R_{*}$. Note that $d \varphi / d r$ is automatically matched at $r=R$ to the exterior solution Eq. (A9). The remaining condition is that the interior field solution at $r=R$,

$$
\varphi(R)=\frac{A}{2} x_{R} g\left(x_{R}\right)+\varphi(0)
$$

matches the exterior solution from Eq. (A11). Figure 11 shows the resulting $\varphi$ profile for different values of $x_{R}$, in comparison with the Newtonian potential $\Psi_{N}$. Again, it is interesting to examine the $x_{R} \gg 1$ and $x_{R} \ll 1$ limits of Eq. (A18). In the former case, we regain the linearized (Newtonian) expectation that the central value is $3 / 2$ of the surface value

$$
\lim _{x_{R} \gg 1} \varphi(0)=-\frac{G \delta M}{\beta R} .
$$

In the opposite, "Vainshtein" limit, the central field like the surface field is independent of $R$ to leading order

$$
\lim _{x_{R} \ll 1} \varphi(0) \approx \varphi(R) \approx-\frac{4 C_{0}}{3 \beta} \frac{G \delta M}{R_{*}} .
$$

The central field value, which determines the potential energy associated with the fluctuation is therefore suppressed by $\left(4 C_{0} / 3\right) R / R_{*}$ from the linearized expectation.

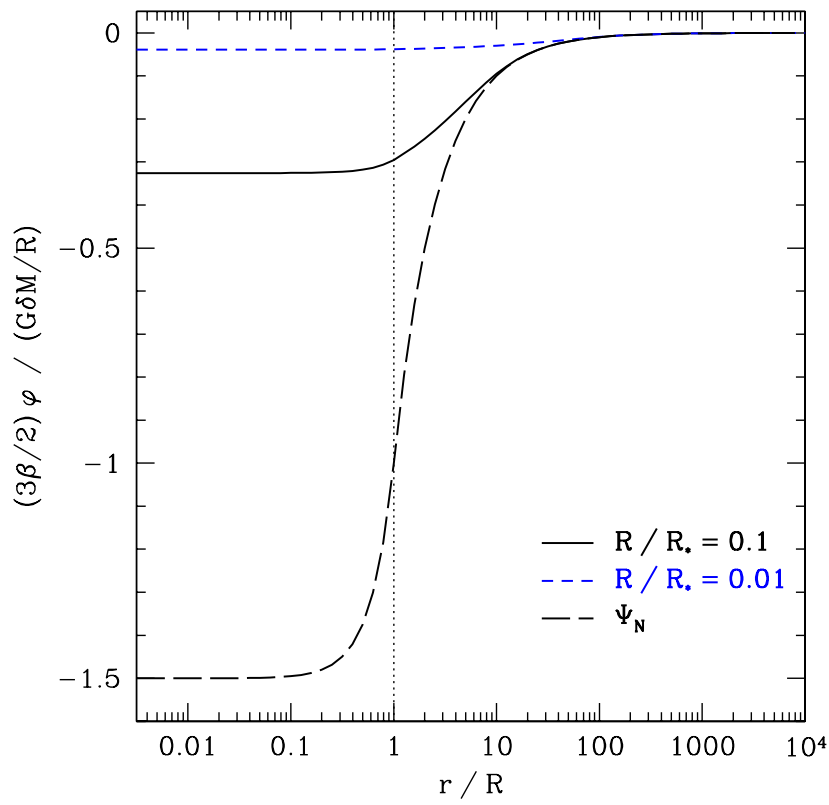

FIG. 11 (color online). Field profile $\varphi(r)$ for a top-hat mass of radius $R$ in units of the Newtonian surface potential $G \delta M / R$, for two values of the Vainshtein radius $R_{*} / R=10,100 R$. $\varphi$ was scaled by $3 \beta / 2$, so that the linearized field solution agrees with the Newtonian potential $\Psi_{N}$ shown as the long-dashed line. 
Moreover, the change in the field interior or near the object, important for forces, is further suppressed by $\left(R / R_{*}\right)^{1 / 2}$. We discuss the consequences of these features of the field profile for virial equilibrium and the potential energy in the following section.

The force suppression can be recast in terms of an effective Newton constant in the Poisson equation for the $\varphi$ field. Note that in the interior solution, the two pieces of the nonlinear term in Eq. (9) combine to form

$$
\left(\nabla^{2} \varphi\right)^{2}-\left(\nabla_{i} \nabla_{j} \varphi\right)^{2}=\frac{2}{3}\left(\nabla^{2} \varphi\right)^{2} .
$$

Since the field equation is then algebraic in $\nabla^{2} \varphi$, one can solve for $\nabla^{2} \varphi$ to obtain

$$
\begin{gathered}
\nabla^{2} \varphi=8 \pi \Delta G_{\mathrm{DGP}}\left(R / R_{*}\right) \delta \rho, \\
\Delta G_{\mathrm{DGP}}(x)=\frac{2}{3 \beta} g(x) x^{2} G=\frac{2}{3 \beta}\left[\sqrt{1+x^{-3}}-1\right] x^{3} G .
\end{gathered}
$$

Hence, in combination with the Newtonian piece, the interior solution can be phrased as possessing an effective $G_{\mathrm{DGP}}=G+\Delta G_{\mathrm{DGP}}$ modification to gravity. Furthermore $x^{-3} \propto \delta$ and so $G_{\text {DGP }}$ is a function of the local density inside the top hat. This relation is specific to the interior of a top hat and is not simply a spherically symmetric approximation as the exterior solution shows. More generally, we can see by taking the derivative of Eq. (A5) that

$$
\nabla^{2} \varphi=8 \pi \Delta G_{\mathrm{DGP}}\left(r / r_{*}\right) \delta \rho(r)+\frac{2 G m(r)}{r^{2}} \frac{d}{d r}\left[x^{2} g(x)\right] .
$$

Note that $\Delta G_{\mathrm{DGP}}, m(r)$ and $r_{*}(r)$ are not local functions of the density field but involve the full interior profile. We shall return to this point in Appendix A 7.

\section{Virial equilibrium}

The virial theorem arises from integrating over space the first moment of the Boltzmann equation, i.e. from the equation of momentum conservation (see e.g. [41], Sec. 4.3). Despite its usual association with potential energy, the virial theorem is inherently a force balance equation and is the collisionless analogue of hydrostatic equilibrium. Thus the virial condition is immune to ambiguities in the definition of potential energy that we shall discuss in Appendix A 4.

The virial theorem reads

$$
W \equiv-\int d^{3} x \rho_{m}(\mathbf{x}) \mathbf{x} \cdot \nabla \Psi=-2 T,
$$

where $W$ receives contribution from the Newtonian gravity of the overdensity, the effective background term, and the brane-bending mode $\varphi$. For a spherically symmetric top hat each contribution to $\Psi$ yields

$$
W_{i}=-3 M \int_{0}^{R} \frac{r^{2} d r}{R^{3}} r \frac{d \Psi_{i}}{d r},
$$

where $\Psi_{i}$ stands for either $\Psi_{N}, \Psi_{\text {eff }}$, or $\Psi_{\varphi} \equiv \varphi / 2$. Thus, any constant offsets in $\Psi_{i}$ do not contribute to $W$. In particular, the constant term in $\varphi$ in the Vainshtein limit of Eq. (A20) and its implied potential energy does not enter into the virial condition.

In the case of the Newtonian contribution, $W_{N}$ defined in this way is given by

$$
W_{N}=-\frac{3}{5} \frac{G M \delta M}{R} .
$$

For the effective contribution of the background $\Psi_{\text {eff }} \propto r^{2}$ and

$$
W_{\text {eff }}=-\frac{4 \pi G}{5}\left[\left(1+3 w_{\text {eff }}\right) \rho_{\text {eff }}+\bar{\rho}\right] M R^{2} .
$$

Note that in our convention, we have included the $\bar{\rho}$ term in $W_{\text {eff }}$ rather than $W_{N}$. Adding the two contributions yields the familiar result [21]:

$$
W_{N}+W_{\text {eff }}=-\frac{3}{5} \frac{G M^{2}}{R}-\frac{4 \pi G}{5}\left(1+3 w_{\text {eff }}\right) \rho_{\text {eff }} M R^{2} .
$$

Since the trace of the potential energy tensor is defined via forces, the $\varphi$ contribution can be described in terms of $\Delta G_{\text {DGP }}$. First, let us examine the exterior region. Using Eq. (A9), we obtain

$$
\frac{d \varphi}{d r}=\frac{4}{3 \beta} \frac{G \delta M}{r^{2}} x^{2} g(x),
$$

which using Eq. (15) becomes

$$
\frac{d \varphi}{d r}=\frac{\Delta G_{\mathrm{DGP}}\left(r / R_{*}\right) \delta M}{r^{2}} .
$$

In the linear regime $r \gg R_{*}, \Delta G_{\mathrm{DGP}}$ then reduces to $(3 \beta)^{-1} G$. Note that in the cosmological context, $\Delta G_{\mathrm{DGP}}$ also has a slow time dependence through $\beta(a)$ [see Eq. (10)].

Using that in the interior $d \varphi / d r \propto r$, we have

$$
W_{\varphi}=-\frac{3 M}{2} \int_{0}^{R} \frac{r^{2} d r}{R^{3}} r \frac{d \varphi}{d r}=-\left.\frac{3 M}{10} \frac{d \varphi}{d \ln r}\right|_{r=R} .
$$

Given that $d \varphi / d r$ at $r=R$ is determined by the exterior solution, we obtain

$$
W_{\varphi}=-\frac{3}{5} \frac{\Delta G_{\mathrm{DGP}} M \delta M}{R},
$$

where $\Delta G_{\mathrm{DGP}}=G_{\mathrm{DGP}}-G$ is the effective gravitational constant for the force modification [Eq. (15)]. Adding all three contributions, we obtain Eq. (31).

Specifically in the collapse calculation, we evaluate the virial condition Eq. (A25) in terms of the kinetic and potential energies per unit mass, written in terms of our 
collapse variable $y(\ln a)$. Defining

$$
E_{0}=\frac{3}{10} M\left(H_{0} R_{i}\right)^{2},
$$

where $R_{i}$ is the initial radius of the perturbation, we obtain

$$
\begin{aligned}
\frac{T}{E_{0}} & =\frac{H^{2}}{H_{0}^{2}}\left(y^{\prime}+a / a_{i}\right)^{2}, \\
\frac{W_{N}}{E_{0}} & =-\Omega_{m} a^{-3}(\delta+1)\left(y+a / a_{i}\right)^{2}, \\
\frac{W_{\varphi}}{E_{0}} & =-\Omega_{m} a^{-3} \frac{\Delta G_{\mathrm{DGP}}\left(R / R_{*}\right)}{G} \delta\left(y+a / a_{i}\right)^{2}, \\
\frac{W_{\mathrm{eff}}}{E_{0}} & =-\left[1+3 w_{\mathrm{eff}}(a)\right] \frac{\rho_{\mathrm{eff}}(a)}{\rho_{\mathrm{cr}, 0}}\left(y+a / a_{i}\right)^{2} .
\end{aligned}
$$

We then define the virial radius as the radius during the collapse at which the virial condition is satisfied. We shall examine approximate techniques for finding this scale through energy conservation in Appendix A 6. As we shall see, with conventional definitions of potential energy, the total energy is not strictly conserved, especially during the initial stages of collapse.

\section{Potential energy definition}

Let us now consider the potential or binding energy of the top-hat mass. For the Newtonian contribution, the potential energy is well defined. By virtue of the Birkhoff theorem, we can view total mass inside the top hat as a Newtonian system in a flat background. We shall see that neither the potential energy contributed by the branebending mode $\varphi$ nor the effective forces of the background expansion are unambiguous to define as both depend on the exterior cosmological context. We therefore follow the convention in the literature in defining them by analogy to the Newtonian contribution.

The Newtonian calculation of potential energy proceeds by replacing the exterior of the top hat with a flat background, the metric analogue to the Newton iron sphere theorem (see [42], Sec. 4). Removing mass shell by mass shell from the outside in, we obtain

$$
U=\int_{0}^{R} \Psi_{\text {tot }}(m(r) ; r) 4 \pi \rho_{m} r^{2} d r,
$$

where $\Psi_{\text {tot }}(m ; r)$ denotes the solution for the total gravitational potential for a top hat with radius $r$ and mass perturbation $m=(r / R)^{3} \delta M$ evaluated at $r$. This is not to be confused with the total potential at a radius $r$ interior to the whole top hat.

Even for the matter contribution, there arises an ambiguity in that the contribution of the background matter density across the top hat is defined only up to a constant $\Psi_{0}$ through $\Psi_{\text {eff }}$ of Eq. (19). In other words, the iron sphere theorem applies directly to forces not potentials and constant offsets do not have any impact on the dynamics. Therefore,

$$
\Psi_{\text {tot }}=\Psi_{N}+\Psi_{\varphi}+\Psi_{\text {eff }}+\Psi_{0} .
$$

This constant $\Psi_{0}$ does not simply introduce a trivial shift in $U$, since even though it is constant across the top hat, it is not necessarily constant as we strip away mass shells. In fact, it is conventional to choose this value to correspond to the result from Newtonian mechanics

$$
\Psi_{0}=-2 \pi G \bar{\rho} R^{2} \equiv-\frac{3}{2} \frac{G \bar{M}}{R},
$$

such that

$$
\Psi_{N}+\Psi_{\text {eff }}+\Psi_{0}=-\frac{G M}{R}+\frac{2 \pi G}{3}\left(1+3 w_{\text {eff }}\right) \rho_{\text {eff }} R^{2} .
$$

The $-G M / R$ piece then corresponds to the Newtonian mechanics result for the potential given the total mass inside the top hat. Using the Birkhoff theorem, this is a valid interpretation of the cosmological case as well.

Note that the $\rho_{\text {eff }}$ piece cannot be properly considered a binding energy, since its contribution cannot be considered without reference to the cosmological background. Even for quintessence models where $\rho_{\text {eff }}$ represents a real energy density, this contribution is supposed to be smooth within its horizon sized Jeans scale regardless of the top-hat collapse and so excising the top hat and placing the mass in a flat background does not strictly make sense.

Nevertheless, under this convention the binding energy from these three components becomes

$$
U_{N}+U_{\mathrm{eff}}+U_{0}=-\frac{3}{5} \frac{G M^{2}}{R}+\frac{2 \pi G}{5}\left(1+3 w_{\mathrm{eff}}\right) M R^{2}
$$

By analogy let us compute the potential energy contribution from $\Psi_{\varphi}$,

$$
U_{\varphi}=\frac{1}{2} \int_{0}^{R} \varphi(m(r) ; r) 4 \pi \rho_{m} r^{2} d r
$$

Again, $\varphi(m ; r)$ denotes the exterior solution of $\varphi$ from Eq. (A11) for a mass $\delta M \rightarrow m$ and radius $R \rightarrow r$. Note that by making this assumption, we are implicitly invoking the Birkhoff theorem where it does not in fact strictly apply. Specifically, as mass shells are stripped away, we ignore the impact of the underdensity left behind outside of the body. As we shall see in Appendix A 7, this underdensity actually changes the interior profile. Nonetheless, since the ambiguity mainly affects the initial stages of collapse when $\delta M \ll M$, it is useful to simply define Eq. (A41) as the binding energy associated with $\varphi$ for energy bookkeeping purposes.

Reexpressing in terms of the dimensionless radius $y=$ $r / R$ (not to be confused with $y$ defined in Sec. III which we shall not use hereafter), we have $m=y^{3} M$, so that $x_{r}=$ $r / r_{*}(m)=R / R_{*}=x_{R}$ is invariant, while $A(m)=$ $y^{2} A(\delta M)$. Therefore, 


$$
\varphi(m ; r)=y^{2} \varphi(\delta M ; R),
$$

where $\varphi(\delta M ; R)$ is the solution for the full mass evaluated at $r=R$ [Eq. (A11) at $x=x_{R}$ ]. This is the same scaling that one obtains for a Newtonian potential, $\Psi(\delta M ; R)=$ $G \delta M / R$ and likewise the potential energy follows the same scaling

$$
U_{\varphi}=\frac{3}{2} M \varphi(\delta M ; R) \int_{0}^{1} y^{4} d y=\frac{3}{10} M \varphi(\delta M ; R) .
$$

The important difference for $U_{\varphi}$ is that $\varphi(\delta M ; R)$, the field profile at $R$, behaves very differently in the linearized and Vainshtein limits

$$
U_{\varphi}= \begin{cases}-\frac{1}{3 \beta} \frac{3}{5} \frac{G M \delta M}{R}, & x_{R} \gg 1, \\ -\frac{2 C_{0}}{5 \beta} \frac{G M \delta M}{R_{*}}, & x_{R} \ll 1 .\end{cases}
$$

Hence, unlike the Newtonian binding energy, $\left|U_{\varphi}\right|$ has a maximum value which is reached asymptotically as $x_{R}=$ $R / R_{*}$ decreases. Thus, while the Newtonian binding energy can become an arbitrarily large fraction of the rest mass energy $\delta M$, the energy in $\varphi$ is limited to a fraction of $G \delta M / \beta R_{*} \sim\left(G \delta M / r_{c}\right)^{2 / 3}$ due to the brane-bending mode interactions regardless of the value of $R$.

\section{Potential energy usage}

Defining a Newtonian based potential energy even though the collapse does not require a Newtonian interpretation is useful for two interrelated reasons. First, it serves as a bookkeeping device if the total energy is conserved during the collapse. Second, it can be used to evaluate the virial condition if it can be simply related to the trace of the potential energy tensor $W$. We examine to what extent these two expectations are satisfied given the field $\varphi$ and the effect of the background expansion.

Let us define the total energy of the perturbation during collapse as

$$
E=T+U .
$$

Taking the time derivative of Eq. (A45) and using the equation of motion of $R(t)$ [Eq. (18)] rewritten using the top-hat profile as

$$
\ddot{R}=-\frac{G_{\mathrm{DGP}} \delta M}{R^{2}}-\frac{4 \pi G}{3}\left[\left(1+3 w_{\mathrm{eff}}\right) \rho_{\mathrm{eff}}+\bar{\rho}\right] R,
$$

we obtain

$$
\frac{d E}{d t}=\frac{3}{5} M \dot{R} \ddot{R}+\frac{\partial U}{\partial R} \dot{R}+\frac{\partial U}{\partial t}=\frac{\partial U}{\partial t} .
$$

Here, the partial derivative $\partial U / \partial t$ receives contributions from evolving quantities in the total potential energy, the sum of Eqs. (A40) and (A43). Since $M$ is conserved, there is no violation of energy conservation for a pure matter system with $\rho_{\text {eff }}=0$ and $\varphi=0$. This is a consequence of adding the $\Psi_{0}$ offset term to reproduce Newtonian mechanics in the matter terms including the background.
On the other hand, both the $\rho_{\text {eff }}$ and $\varphi$ contributions have explicit time evolution across a Hubble time. Note that violation of energy conservation due to evolution of $\rho_{\text {eff }}$ also applies to dark energy models where $w_{\text {eff }} \neq-1$. In the $\varphi$ term given by Eq. (A43), $\beta$ evolves with the expansion, and as long as $\delta$ is not much greater than 1 , $\delta M$ also evolves during collapse. More generally, these effects occur whenever the modification to the background (due to modified gravity or the presence of dark energy) does not match that of the perturbations. We return to the impact of energy nonconservation in Appendix A 6. Noncon-

servation of the Newtonian total energy defined in Eq. (A45) does not mean a violation in covariant conservation of energy and momentum.

In order to use the potential energy to assist the evaluation of the virial theorem, we must relate $U$ to the trace of the potential energy tensor $W$. It is well known that for a potential satisfying $\Psi(r) \propto r^{\alpha}$ and for which the interior solution is $\Psi_{\text {int }}(r<R)=\Psi_{\text {ext }}(m(r) ; r), \quad W_{i}=-\alpha U_{i}$. Hence, for potentials satisfying this condition, the potential energy determined by the potential itself is of the same order as the trace of the potential energy tensor defined by the forces. This holds for the Newtonian contribution, where we have $U_{N}=W_{N}$, and the effective background contribution, which satisfies $U_{\text {eff }}=-W_{\text {eff }} / 2$. For the brane-bending mode, the potential is no longer a pure power law and the distinction between $U$ and $W$ leads to interesting consequences in the Vainshtein limit.

Note that in this $x_{R} \ll 1$ limit, the potential energy $U_{\varphi}$ is dominated by the constant term in Eq. (A13), while the contribution from the $x^{1 / 2}$ part of the profile which determines forces is much smaller. Correspondingly, the assumption that the trace of the potential energy tensor $W$ is of order the potential energy $U$ is not valid for the $\varphi$ contribution, as the change in the potential across the body is much smaller than the potential depth itself. The relationship between $W_{\varphi}$ and $U_{\varphi}$ follows from Eqs. (A32) and (A43):

$$
W_{\varphi}=-\left.\frac{d \ln \varphi}{d \ln r}\right|_{r=R} U_{\varphi}=-\frac{d \ln U_{\varphi}}{d \ln R} U_{\varphi} .
$$

In the $R \gg R_{*}$ limit, $\varphi \propto r^{-1}$ and $W_{\varphi}=U_{\varphi}$ as usual, but in the $R \ll R_{*}$ limit, $\varphi \approx$ const and the trace of the potential tensor is highly suppressed compared to the potential energy. Figure 12 shows $U_{\varphi}$ and $W_{\varphi}$ as a function of the overdensity $\delta=\delta \rho / \bar{\rho}$ of the perturbation. Note that if we were to interpret the Vainshtein effect as simply a modification of $G$, we would infer the wrong energy condition at virialization. We discuss this issue in the next section.

\section{Misestimating virial overdensity}

Most commonly, the virial condition Eq. (29) is evaluated using energy conservation. At virialization, 
FABIAN SCHMIDT, WAYNE HU, AND MARCOS LIMA

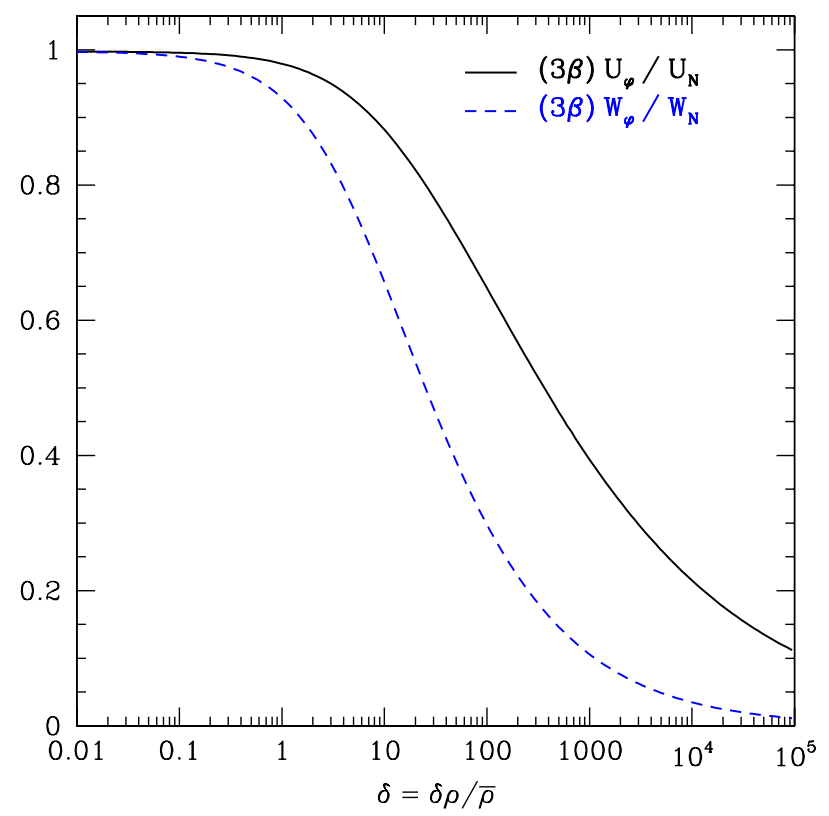

FIG. 12 (color online). Scaled brane-bending mode binding energy $3 \beta \times U_{\varphi}$ in units of the Newtonian binding energy $U_{N}=$ $-3 G M \delta M / 5 R$ (black solid) as a function of the overdensity $\delta$ for a spherical top-hat mass at $z=0$ in the sDGP cosmology. The blue dashed line shows the trace of the potential energy tensor $(3 \beta) \times W_{\varphi}$ used in the virial theorem, Eq. (A32), again with respect to the Newtonian value $W_{N}=U_{N}$.

$$
T\left(R_{\text {vir }}\right)=-\frac{1}{2} W\left(R_{\text {vir }}\right)
$$

and the total energy $E=T\left(R_{\text {vir }}\right)+U\left(R_{\text {vir }}\right)=U\left(R_{\text {vir }}\right)-$ $W\left(R_{\text {vir }}\right) / 2$. Since at turnaround $\left(R=R_{\text {ta }}\right)$ the kinetic energy vanishes, we have $E=U\left(R_{\mathrm{ta}}\right)$. Assuming energy conservation, we obtain

$$
U\left(R_{\mathrm{ta}}\right)-U\left(R_{\mathrm{vir}}\right)+\frac{1}{2} W\left(R_{\mathrm{vir}}\right)=0 .
$$

By further assuming a relationship between the potential energy $U$ and the trace of the potential tensor $W$, we can solve for $R_{\mathrm{vir}} / R_{\mathrm{ta}}$. There are therefore two ways by which this association can go wrong: if $E$ is not conserved and if an incorrect relation between $U$ and $W$ is employed.

Let us begin by examining the first issue. Energy is not strictly conserved in any model where either $w_{\text {eff }} \neq-1$ (including quintessence), modifications to gravity are time variable, or force modifications are only generated by the perturbed mass $\delta M$.

Evaluating Eq. (A45) during collapse, we found that in the effective dark energy model QCDM [which has the same $H(z)$ as sDGP], energy conservation is violated by $\sim 3 \%$ from turnaround to collapse. While the violation of energy conservation in dark energy models thus seems to be minor, it does influence the virial overdensity due to the sensitivity of $\Delta_{\text {vir }}$ to $R_{\text {vir }}$ and $a_{\text {vir }}$. Figure 13 shows $\Delta_{\text {vir }}$ as function of a constant dark energy equation of state $w_{\text {eff }}$ and $\Omega_{\text {eff }}=0.741$, determined by evaluating the virial
PHYSICAL REVIEW D 81, 063005 (2010)

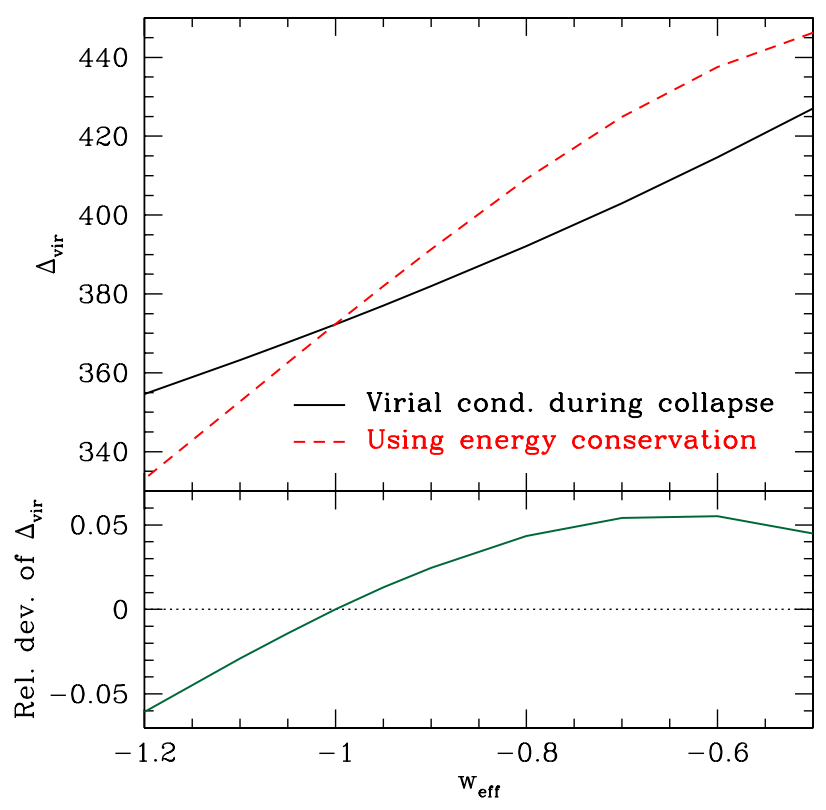

FIG. 13 (color online). Misestimation of the virial overdensity $\Delta_{\text {vir }}$ in quintessence dark energy models. $\Delta_{\text {vir }}$ as defined in Sec. III C for collapse at $a_{0}=1$, as a function of the dark energy equation of state parameter $w_{\text {eff }}=$ const and $\Omega_{\text {eff }}=0.741$ i.e. for standard gravitational forces (top panel). The solid line shows $\Delta_{\text {vir }}$ determined by evaluating the virial condition during collapse, the approach adopted here, while the dashed line shows the usual calculation using standard energy conservation as described in Appendix A 6. Both calculations agree for $w=$ -1 , i.e. $\rho_{\text {eff }}=$ const. The lower panel shows the relative deviation between the two, $\left(\Delta_{\text {vir }}^{\text {std }}-\Delta_{\text {vir }}\right) / \Delta_{\text {vir }}$.

condition during collapse (our approach) and using energy conservation (e.g., [21]). While both approaches agree for $w_{\text {eff }}=-1$ as expected, there are clear differences as soon as $w_{\text {eff }} \neq-1$. Note that these differences are of the same order as the difference $\Delta_{\text {vir }}\left(w_{\text {eff }}\right)-\Delta_{\text {vir }}\left(w_{\text {eff }}=-1\right)$.

Our approach does not rely on exact energy conservation, and one might thus expect the $\Delta_{\text {vir }}$ obtained in this way would lead to a better match to observables. Since quantities such as the mass function are typically simply fit to simulations with a given definition of overdensity, this is in part an issue of semantics. However, use of a more physically motivated scaling might lead to a more universal form for the mass function or one that scales more simply with parameters of the theory. It would however be necessary to compare with $N$-body simulations of $w_{\text {eff }} \neq$ -1 dark energy models, and that is beyond the scope of this work. Here, we simply note that the dependence on $w_{\text {eff }}$ of $\Delta_{\text {vir }}$ determined using our approach is smaller than that of the usual definition and so it would predict a more universal scaling with a fixed overdensity than the standard approach.

The deviations from $E=$ const are much larger in the DGP modified force case, where evolving forces and $\delta M$ lead to stronger evolution of $E$. Here, differences in the total energy between turnaround and collapse are $10-15 \%$. 
Thus, the choice of procedure for determining $\Delta_{\text {vir }}$ becomes even more important. Again, we found that our approach (greatly) reduces the dependence of $\Delta_{\text {vir }}$ on the evolution of the modified forces.

Finally, we consider the effect of assuming an incorrect relation between $U$ and $W$ in Eq. (A50). For example, it is tempting to just set $U_{\varphi}=W_{\varphi}=\Delta G_{\mathrm{DGP}} / G \times W_{N}$, i.e., ignoring the $R$-independent term in $U_{\varphi}$. However, since $\Delta G_{\text {DGP }} \rightarrow 0$ in the late stages of collapse $(\delta \rightarrow \infty), U_{\varphi}$ would erroneously be set to 0 in this approximation. For $\beta$ of order unity, this leads to apparent violations of energy conservation at the level of $30 \%$. When using energy conservation in the presence of the $\varphi$ field, it is thus crucial to take into account the differences between $U_{\varphi}$ and $W_{\varphi}$.

\section{Compensated top-hat profile}

Finally we study one example of a density profile beyond the pure top hat, the compensated top-hat profile. In the cosmological context, a collapsing top-hat perturbation sweeps out "empty space" and in fact has the following density profile:

$$
\rho(r)-\bar{\rho}= \begin{cases}\bar{\rho} \delta, & r \leq R, \\ -\bar{\rho}, & R<r \leq R_{e}, \\ 0, & r>R_{e},\end{cases}
$$

where $R_{e}=R_{i} a / a_{i}$ is the physical radius today corresponding to the radius of the perturbation at an early time $a_{i}$ when $\delta \ll 1$. We continue to call the total mass and mass perturbation enclosed at $R$ as $M$ and $\delta M \equiv m(R)$, respectively.

In terms of the scaled radial coordinate $y \equiv r / R$, the full description for the enclosed mass perturbation becomes

$$
m(r)= \begin{cases}\delta M y^{3}, & y \leq 1, \\ M\left[1-\left(y / y_{e}\right)^{3}\right], & 1<y \leq y_{e} \\ 0, & y>y_{e}\end{cases}
$$

Importantly, forces at a given radius only depend on the enclosed mass $m(r)$ through Eq. (A5). Given that for $r<$ $R$, the enclosed mass of the compensated and uncompensated top hat are the same, compensation has no impact on the interior dynamics of collapse. Likewise, from the definition of the potential energy tensor Eq. (A26), we see then that $W_{\varphi}$ is unchanged from that of the pure top-hat profile, and hence the virial condition is unmodified.

Naively, one might assume that as long as $\delta \gg 1$, the compensation has little effect on the binding energy or gravitational potential $\Psi$ in the interior as well, but we shall see that this is not necessarily so for the branebending contribution due to the Vainshtein suppression.

In the exterior $(y>1)$ forces from $\varphi$ are modified by the compensation as

$$
\begin{aligned}
\frac{d \varphi}{d y} & =R \frac{d \varphi}{d r} \\
& =\left(\frac{2}{3 \beta} \frac{G \delta M}{R}\right) \frac{2 y}{y_{*}^{3}}\left[\sqrt{\left(\frac{y_{*}}{y}\right)^{3} \frac{y_{e}^{3}-y^{3}}{y_{e}^{3}-1}+1}-1\right] .
\end{aligned}
$$

In the limit $y_{e} \gg y \geq 1$, the forces are the same as in the uncompensated profile (see Fig. 14). Since $y_{e}^{3}=1+\delta$, forces are unchanged near the body, $y \sim 1$, as long as $\delta \gg$ 1 . Even for $\delta<1$, this modification does not introduce any physical effect on the collapse since there is no mass in the exterior region $1<y<y_{e}$ that could be moved by the modified forces. Hence an initial top-hat profile will remain a top hat during collapse.

Now let us look at the effect of compensation near the Vainshtein scale of the mass, $y_{*} \equiv R_{*} / R$. Note that

$$
\frac{y_{*}^{3}}{y_{e}^{3}}=\epsilon \frac{\delta}{1+\delta},
$$

where $\epsilon^{-1}$ is the density threshold beyond which the Vainshtein mechanism operates as defined in Eq. (27). Unless this density threshold also satisfies $\epsilon^{-1} \gg 1$, compensation effects will change how the profile saturates, since $y_{*}$ will be comparable to $y_{e}$. Correspondingly in Eq. (A53), both $\delta \gg 1$ and $\epsilon \ll 1$ are necessary for $d \varphi / d y$ to recover the uncompensated result at the Vainshtein scale $y_{*}$. Given that the top-hat $\varphi$ profile within $R_{*}$ is controlled by its value at $R_{*}$, we expect the $\varphi$ profile itself to be modified near the body by the density compensation unless $\epsilon \ll 1$.

More specifically, let us consider the linearized $\left(y_{*} \ll y\right)$ and Vainshtein $\left(y_{*} \gg y\right)$ limits as before. First note that even the Newtonian force $d \Psi_{N} / d r$ for the compensated top hat at $y>1$ is modified as

$$
R \frac{d \Psi_{N}}{d r}=\frac{G m(r)}{R} y^{-2}=\frac{G \delta M}{R} \frac{y_{e}^{3}-y^{3}}{y_{e}^{3}-1} y^{-2} .
$$

In the linearized limit, Eq. (A53) reduces to

$$
\lim _{y \gg y_{*}} \frac{d \varphi}{d r}=\frac{2}{3 \beta} \frac{d \Psi_{N}}{d r}
$$

as expected. In the Vainshtein limit of $y_{*} \gg y(>1)$, and in which case $y \ll y_{e}$ as well, the force contributions take the form

$$
\lim _{y \ll y_{*}} \frac{d \varphi}{d y}=\left(\frac{2}{3 \beta} \frac{G \delta M}{R}\right) \frac{2}{\sqrt{y}} y_{*}^{-3 / 2}\left(\frac{y_{e}^{3}-y^{3}}{y_{e}^{3}-1}\right)^{1 / 2} .
$$

We thus recover the leading $r^{-1 / 2}$ behavior of the force in this limit. Figure 14 shows $d \varphi / d y$ as function of $y$ for different values of $y_{*}=R_{*} / R$. Note that unlike the Newtonian case, the force given by $d \varphi / d y$ differs from that of an equivalent top hat with radius $r$ and enclosed mass fluctuation $m(r)$ for any $r>R$. This behavior cannot be described by a simple $G_{\mathrm{DGP}}(\delta)$ parametrization. 


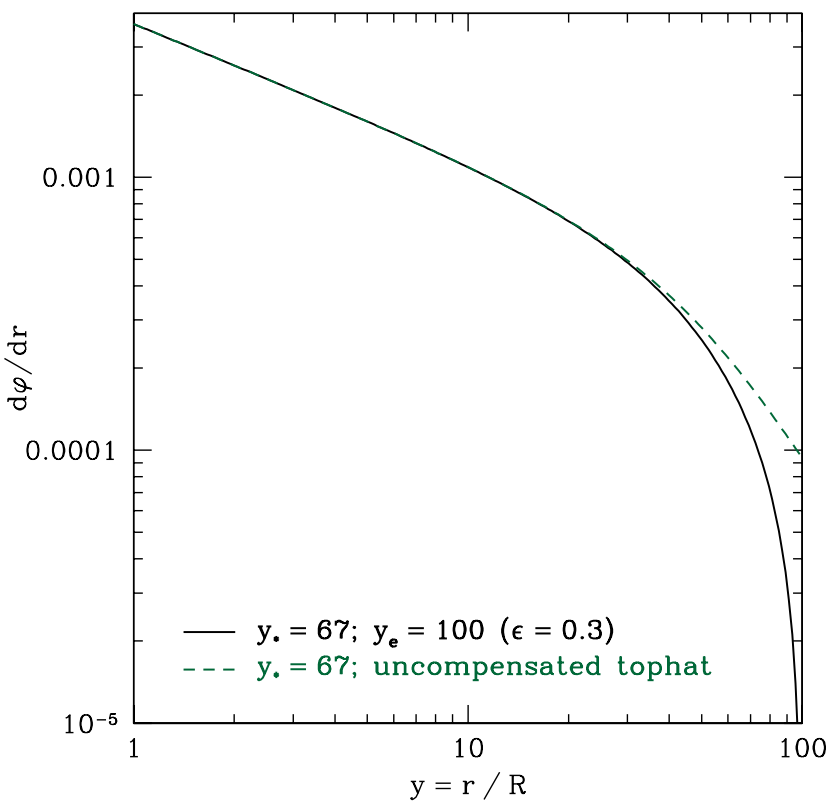

FIG. 14 (color online). Field gradient $d \varphi / d r$ for a compensated top-hat profile (solid) in units of $2 /(3 \beta) G \delta M / R^{2}$ as a function of $y=r / R \cdot y_{e}=R_{e} / R$ is set to 100 (corresponding to $\delta=10^{6}$ ), and $y_{*}=R_{*} / R=67$, corresponding to $\epsilon=0.3$ valid for the sDGP model. The dashed line shows $d \varphi / d r$ for the same top-hat mass but with uncompensated profile. Near $R$, force modifications from $\varphi$ are not affected by compensation.

Next, as with the pure top hat, we can solve for the whole field profile given the boundary condition $\varphi\left(R_{e}\right)=0$ by integrating

$$
\varphi(r>R)=-\int_{r / R}^{y_{e}} \frac{d \varphi}{d y} d y .
$$

We then obtain

$$
\varphi(y)=-\left(\frac{2}{3 \beta} \frac{G \delta M}{R}\right) \frac{y_{e}^{2}}{y_{*}^{3}}\left[\frac{y^{2}}{y_{e}^{2}}-1+F(1, \epsilon)-F\left(\frac{y}{y_{e}}, \epsilon\right)\right],
$$

where

$$
F(x, \epsilon) \equiv 4 \sqrt{\epsilon x}_{2} F_{1}\left[-1 / 2,1 / 6 ; 7 / 6 ; x^{3}\left(1-\epsilon^{-1}\right)\right] .
$$

Note that this is a slightly different hypergeometric function than that in the pure top-hat field solution. Figure 15 shows $\varphi(y)$ vs $y$ for a fixed overdensity $\delta=10^{6}$ for two different values of $y_{*}$ (or, equivalently, $\epsilon$ ). Again, we obtain the expected scaling in the limiting cases. For the linear limit $y \gg y_{*}$, we have

$$
\begin{aligned}
\lim _{y \gg y_{*}} \varphi(y) & =\left(\frac{2}{3 \beta} \frac{G \delta M}{R}\right)\left[-\frac{y^{2}}{2 \delta}-\frac{\delta+1}{y \delta}+\frac{3}{2} \frac{(\delta+1)^{2 / 3}}{\delta}\right] \\
& =\frac{2}{3 \beta} \Psi_{N},
\end{aligned}
$$

proportional to the Newtonian potential for a compensated

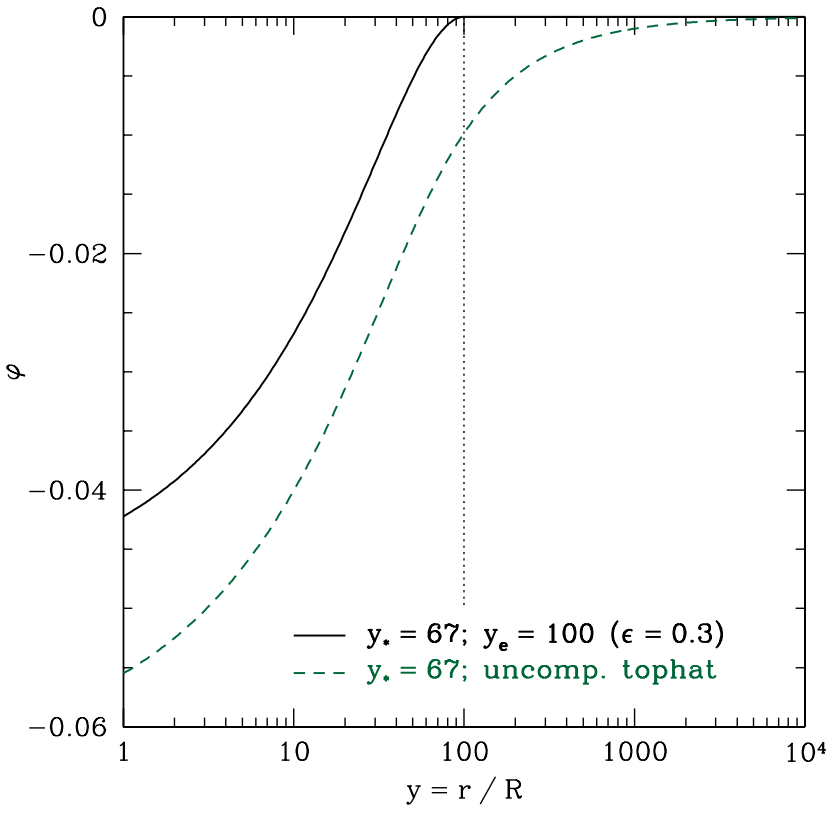

FIG. 15 (color online). The exterior $\varphi$ profile in units of $2 /(3 \beta) G \delta M / R$ as function of the scaled radius $y$ for a compensated top-hat profile (solid). $y_{e}=100$ and $y_{*}=67$ as in Fig. 14. The field goes to 0 at $y=y_{e}$ (dotted vertical line). The dashed line shows the profile for an uncompensated top hat with the same mass and radius. Note that the $\varphi$ field for the two profiles differs at all radii, reflecting the fact that $\varphi(r)$ is not simply determined by the enclosed mass at $r$.

top hat with the same boundary condition, $\Psi_{N}\left(R_{e}\right)=0$. As expected, for $\delta \gg 1$ the profile matches the uncompensated case until $y$ approaches $y_{e}$.

In the Vainshtein limit, $y_{*} \approx \epsilon^{1 / 3} y_{e} \gg 1$ and the profile becomes

$$
\lim _{y \ll y_{*}} \varphi(y)=-\left(\frac{4}{3 \beta} \frac{G \delta M}{R_{*}}\right)\left[C_{\epsilon}-2 \sqrt{y / y_{*}}\right]
$$

where

$$
C_{\epsilon}=\frac{F(1, \epsilon)-1}{2 \epsilon^{2 / 3}} .
$$

In the limit $\epsilon \rightarrow 0, C_{\epsilon}=C_{0}$, and the profile returns to the uncompensated form of Eq. (A20). In Fig. 16 we show $C_{\epsilon} / C_{0}$ the reduction in the surface potential $\varphi(R)$ due to the compensation in the Vainshtein limit.

One can also define an alternate definition of the binding energy of the perturbation $\delta M$. Suppose that we again define the binding energy $U_{\varphi}$ as in Eq. (A41) by removing shell by shell of the mass to $R_{e}$. However, in this case we properly account for the impact of the exterior. As each shell is removed to $R_{e}$, it fills in the mass deficit so that $R_{e}$ decreases in such a way as to keep $y_{e}=R_{e} / R$ constant.

Thus, the only part of Eq. (A59) which scales nontrivially is the prefactor $\delta M / R \propto R^{2}$ as in the case of the pure top hat. Equation (A43) strictly holds for the compensated 
top hat

$$
U_{i}=\frac{3}{10} M \Psi_{i}(\delta M, R)
$$

for the Newtonian and $\varphi$ contributions. In particular, in the Vainshtein regime, the modification in $U_{\varphi}$ from the pure top hat is given by the constant piece in the profile which scales with $\epsilon$ as in Fig. 16.

In fact, this derivation unlike that for the pure top hat is fully self-consistent in that the form of the profile, including the background contribution, is self-similar as the shells are removed. Furthermore, the end result is a compensated top hat of $R \rightarrow 0$, i.e. an unperturbed universe with no source to $\varphi$. In this view, the binding energy associated with $\delta M$ is the energy required to eliminate the mass perturbation rather than the mass.

Unfortunately, this definition still does not fully resolve the ambiguities associated with the potential energy definition. We still need to account for the potential energy due to the background density. In particular, in this definition the Newtonian binding energy also only accounts for the perturbation and hence using it in the definition of total energy would not obey strict energy conservation during the collapse, especially for $\delta<1$. We have seen that in the literature, the definition of potential energy is mainly used in conjunction with energy conservation to simplify the calculation of the virial radius. In this context, the original definition of binding energy as that of a pure top hat with no contribution from the exterior is more useful. In this case, it is important to keep in mind however that modified

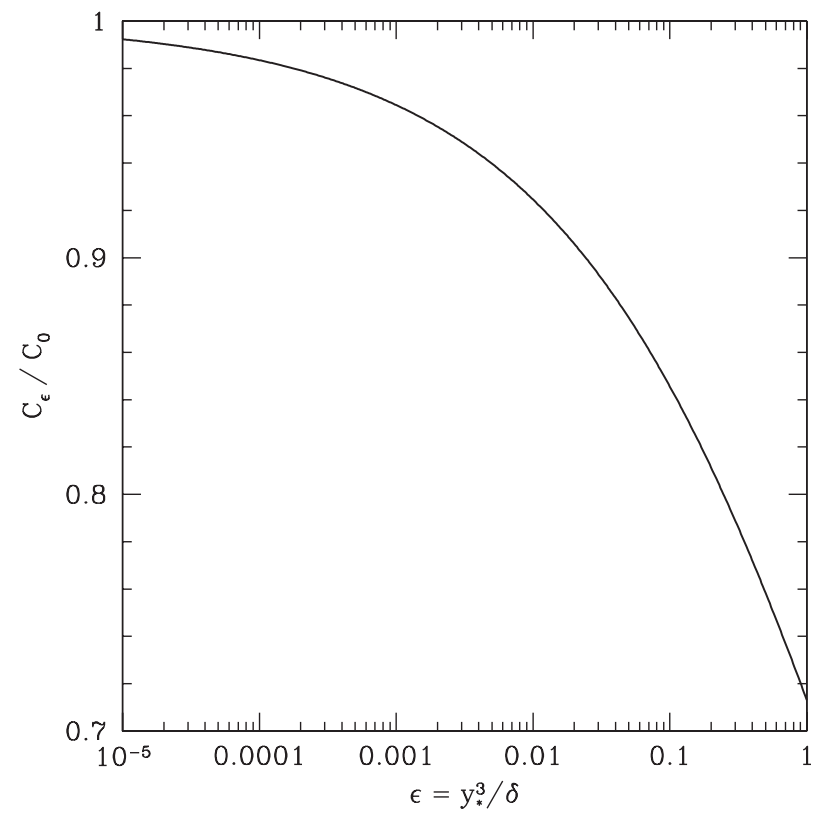

FIG. 16. Reduction in the surface potential due to compensation in the Vainshtein limit, $C_{\epsilon} / C_{0}$, as a function of $\epsilon=y_{*}^{3} / \delta$. In the limit $\epsilon \ll 1, y_{*} \ll y_{e}$ and the compensation has little effect on the $\varphi$ profile. This quantity also controls the reduction in binding energy.

forces as well as evolving dark energy density generally imply a violation of energy conservation (Appendix A 5).
[1] A. G. Riess et al., Astrophys. J. 659, 98 (2007).

[2] M. Kowalski et al., arXiv:0804.4142.

[3] J. Dunkley et al., Astrophys. J. Suppl. Ser. 180, 306 (2009).

[4] T. Giannantonio et al., Phys. Rev. D 74, 063520 (2006).

[5] D. Pietrobon, A. Balbi, and D. Marinucci, Phys. Rev. D 74, 043524 (2006).

[6] A. Lue, R. Scoccimarro, and G. D. Starkman, Phys. Rev. D 69, 124015 (2004).

[7] H. Oyaizu, M. Lima, and W. Hu, Phys. Rev. D 78, 123524 (2008).

[8] F. Schmidt, M. Lima, H. Oyaizu, and W. Hu, Phys. Rev. D 79, 083518 (2009).

[9] F. Schmidt, Phys. Rev. D 80, 043001 (2009).

[10] G. Dvali, G. Gabadadze, and M. Porrati, Phys. Lett. B 485, 208 (2000).

[11] C. Deffayet, Phys. Lett. B 502, 199 (2001).

[12] A. Nicolis and R. Rattazzi, J. High Energy Phys. 6 (2004) 59.

[13] K. Koyama and R. Maartens, J. Cosmol. Astropart. Phys. 1 (2006) 16.

[14] K. C. Chan and R. Scoccimarro, Phys. Rev. D 80, 104005 (2009).
[15] G. Dvali, S. Hofmann, and J. Khoury, Phys. Rev. D 76, 084006 (2007).

[16] A. Nicolis, R. Rattazzi, and E. Trincherini, Phys. Rev. D 79, 064036 (2009).

[17] A. Cooray and R. Sheth, Phys. Rep. 372, 1 (2002).

[18] P. J. E. Peebles, The Large-Scale Structure of the Universe (Princeton University Press, Princeton, NJ, 1980), p. 435 (Research supported by the National Science Foundation).

[19] O. Lahav, P. B. Lilje, J. R. Primack, and M. J. Rees, Mon. Not. R. Astron. Soc. 251, 128 (1991).

[20] L. Wang and P. J. Steinhardt, Astrophys. J. 508, 483 (1998).

[21] C. Horellou and J. Berge, Mon. Not. R. Astron. Soc. 360, 1393 (2005).

[22] S. Basilakos, J.C.B. Sanchez and L. Perivolaropoulos, Phys. Rev. D 80, 043530 (2009).

[23] W. Fang et al., Phys. Rev. D 78, 103509 (2008).

[24] M. A. Luty, M. Porrati, and R. Rattazzi, J. High Energy Phys. 09 (2003) 029.

[25] K. Koyama, Classical Quantum Gravity 24, R231 (2007).

[26] F. Schmidt, Phys. Rev. D 80, 123003 (2009).

[27] L. Lombriser, W. Hu, W. Fang, and U. Seljak, Phys. Rev. D 80, 063536 (2009). 
[28] K. Koyama and F. P. Silva, Phys. Rev. D 75, 084040 (2007).

[29] J. Khoury and M. Wyman, Phys. Rev. D 80, 064023 (2009).

[30] R. Scoccimarro, Phys. Rev. D 80, 104006 (2009).

[31] R. Sheth and B. Tormen, Mon. Not. R. Astron. Soc. 308, 119 (1999).

[32] W. Hu and A. V. Kravtsov, Astrophys. J. 584, 702 (2003).

[33] J. F. Navarro, C.S. Frenk, and S. D. M. White, Astrophys. J. 490, 493 (1997).

[34] J. S. Bullock et al., Mon. Not. R. Astron. Soc. 321, 559 (2001).

[35] A. Vikhlinin et al., Astrophys. J. 692, 1060 (2009).
[36] E. Rozo et al., Astrophys. J. 708, 645 (2010).

[37] A. Mantz, S. W. Allen, D. Rapetti, and H. Ebeling, arXiv:0909.3098.

[38] R. E. Smith et al., Mon. Not. R. Astron. Soc. 341, 1311 (2003).

[39] L. Hui, A. Nicolis, and C. Stubbs, Phys. Rev. D 80, 104002 (2009).

[40] W. Hu, Nucl. Phys. B, Proc. Suppl. 194, 230 (2009).

[41] J. Binney and S. Tremaine, Galactic Dynamics (Princeton University Press, Princeton, NJ, 1987).

[42] P. Peebles, Principles of Physical Cosmology (Princeton University Press, Princeton, NJ, 1993). 